\title{
The Aza-Type B Photochemical Rearrangement; the Role of a Second Carbonyl in Heterocyclic Photochemistry. Mechanistic and Exploratory Organic Photochemistry ${ }^{1,2}$
}

SUPPORTING INFORMATION

Howard E. Zimmerman* and Oleg D. Mitkin

Department of Chemistry, University of Wisconsin-Madison, Madison, Wisconsin 53706

zimmerman@chem.wisc.edu

\section{CONTENTS of the Supporting Material}

General Procedures

NMR Spectra

S2-S35

Computational Detail

S36-S41

General Procedures. All reactions were performed under an atmosphere of dry nitrogen. Melting points were determined in open capillaries and are uncorrected. Column chromatography was performed on silica gel (Aldrich, $60 \AA$, 200-400 mesh, or Silica gel 60 Geduran® $35-75 \mu \mathrm{m}$ ) mixed with Sylvania 2282 phosphor and slurry packed into quartz columns to allow monitoring with a hand-held UV lamp. All solvents were additionally purified and dried by standard techniques. ${ }^{1} \mathrm{H}$ and ${ }^{13} \mathrm{C}$ NMR spectra were recorded at $300 \mathrm{MHz}$ and $75 \mathrm{MHz}$ respectively, and are reported in ppm downfield from tetramethylsilane. Solution photolyses were carried out in an immersion reactor 
with water-cooled jacket using a $400 \mathrm{~W}$ medium-pressure mercury lamp equipped with a $5 \mathrm{~mm}$ filter of circulating $0.4 \mathrm{M}$ solution of $\mathrm{CuSO}_{4}$ unless stated otherwise. All solutions were purged with deoxygenated nitrogen for $1 \mathrm{~h}$ both prior to and during photolysis. 
$000^{\circ} 0$

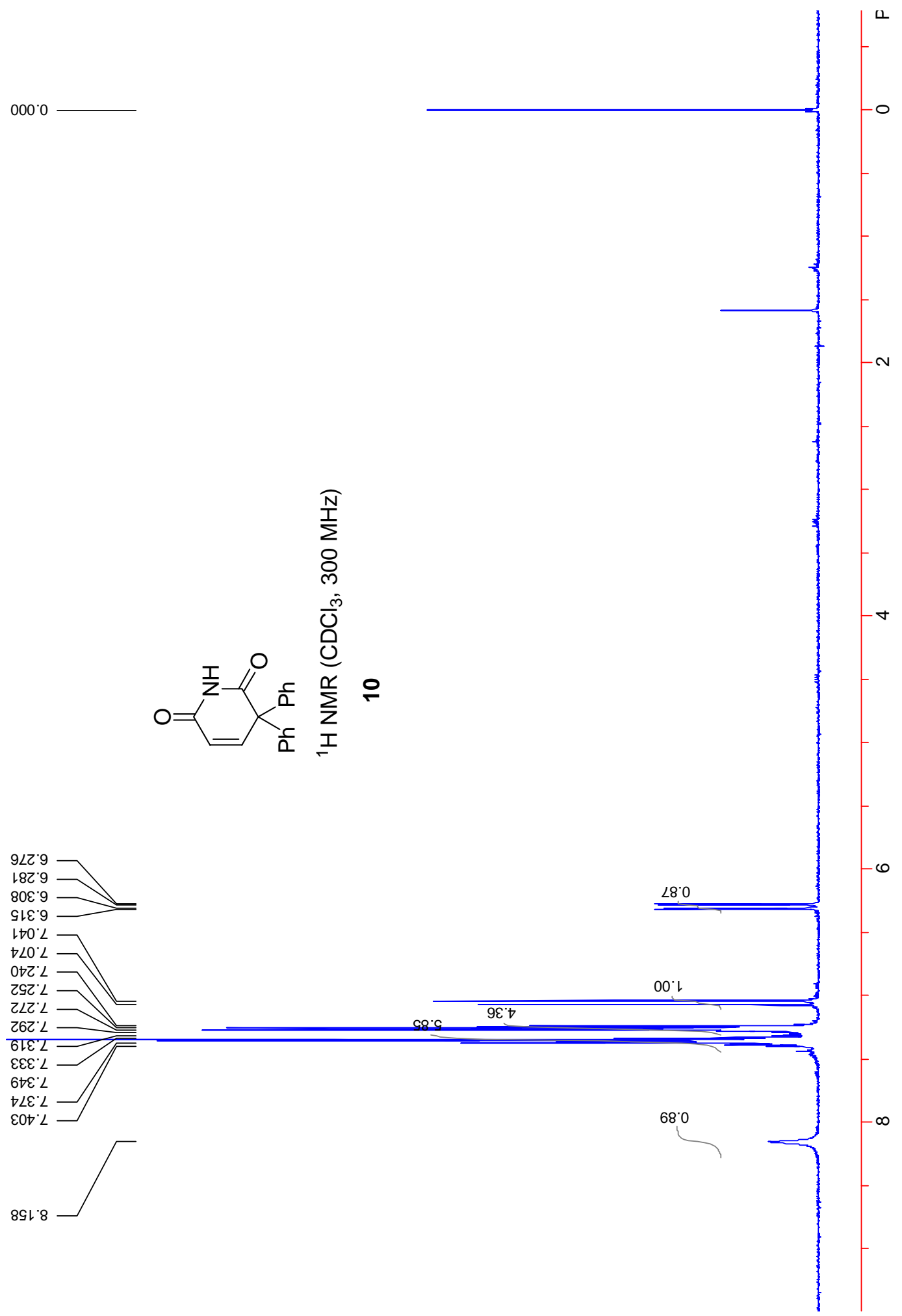




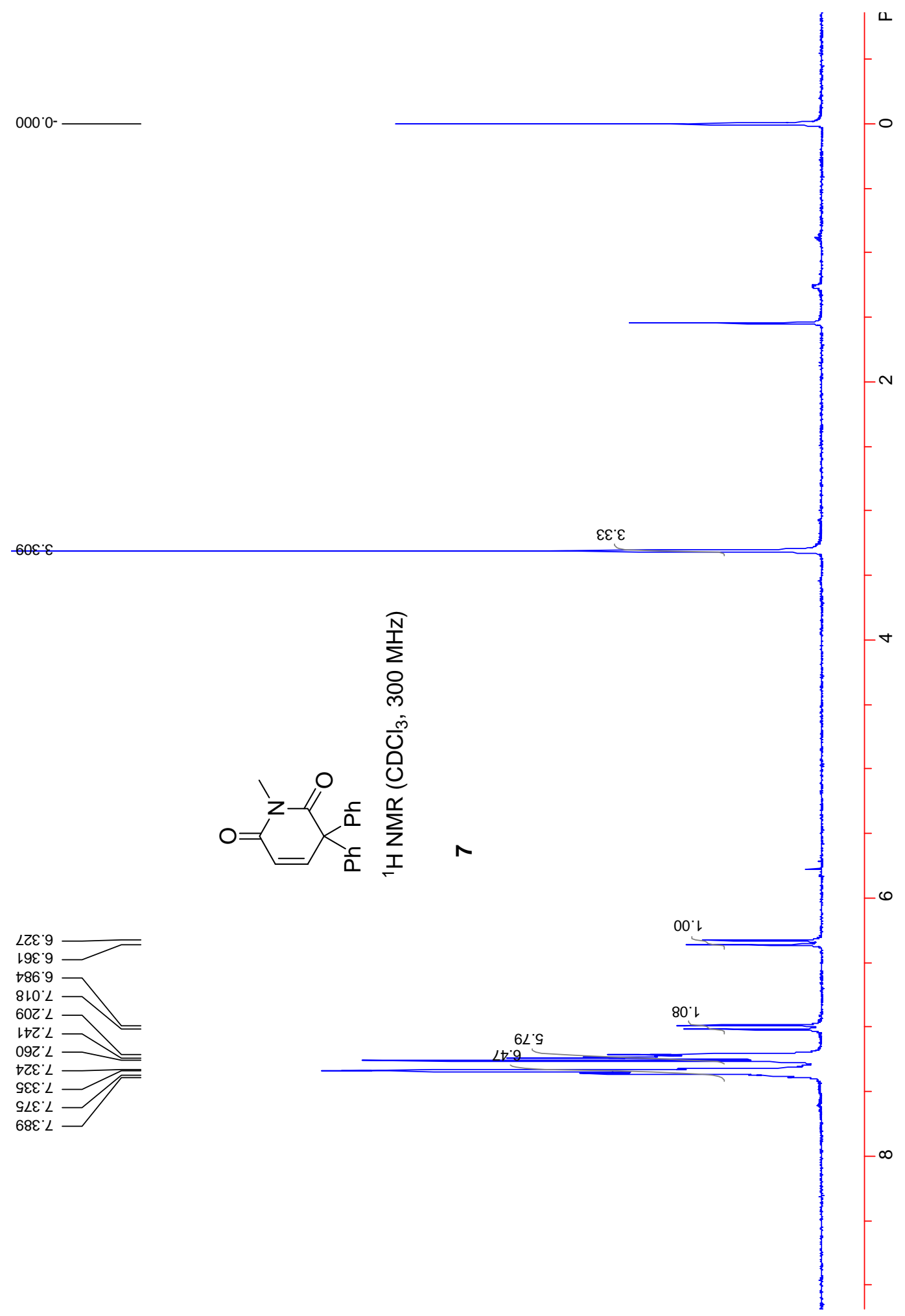


$20 t^{\circ} 92$

เ92 69

${ }^{\circ} 6 \varepsilon^{\circ} 9 L$

ㄴ18.9L

$\varepsilon E Z \angle L$

$\varepsilon 206$ t

IS8: LZL

$+0+821$
$I \angle 9^{\circ} 821$

$\varepsilon 88^{\circ} 0$ th

$2 \angle 0^{\circ} 8 t 1$

เ8เ๋9เ -

†๑t' $\varepsilon \angle t$
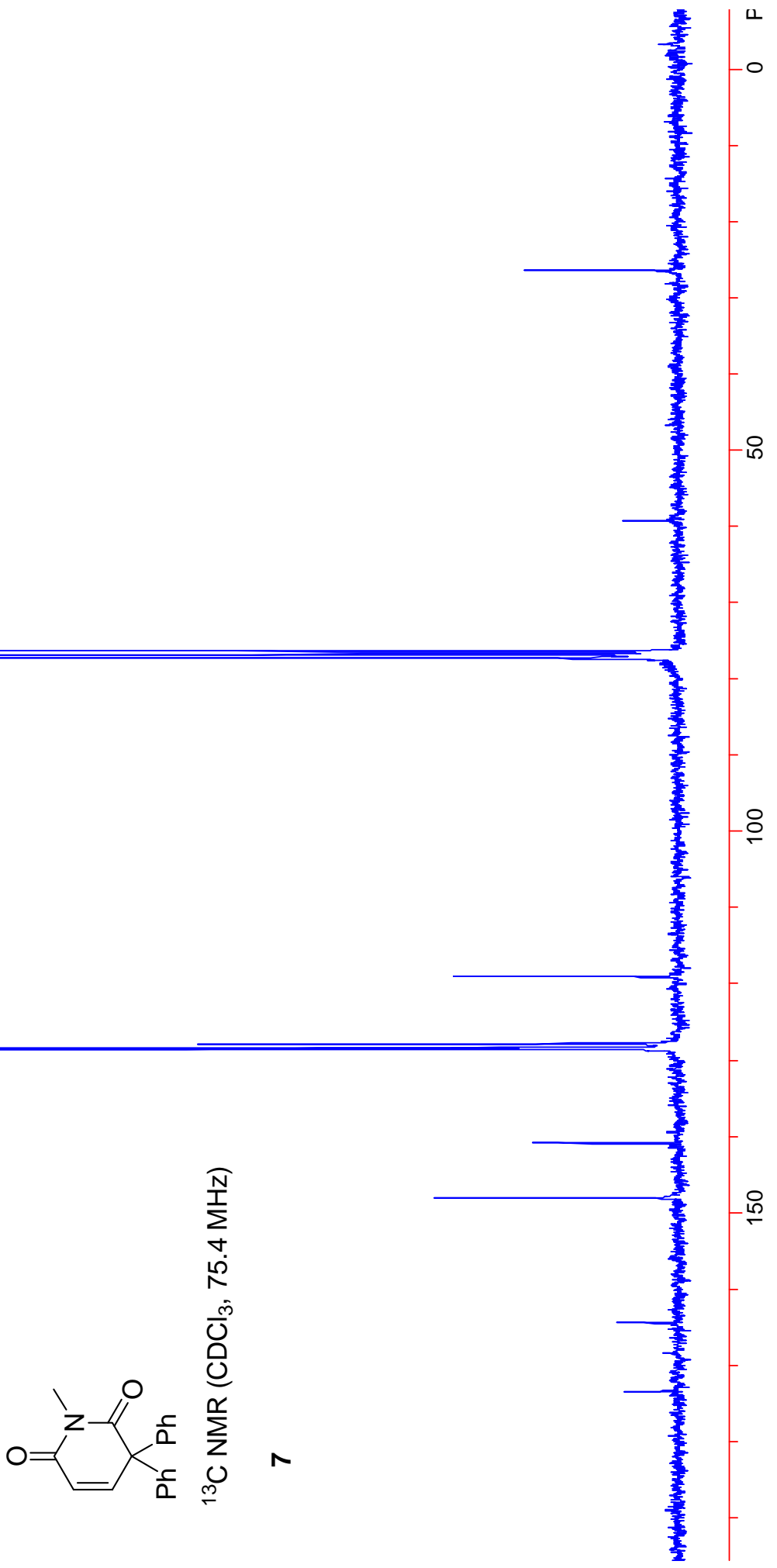


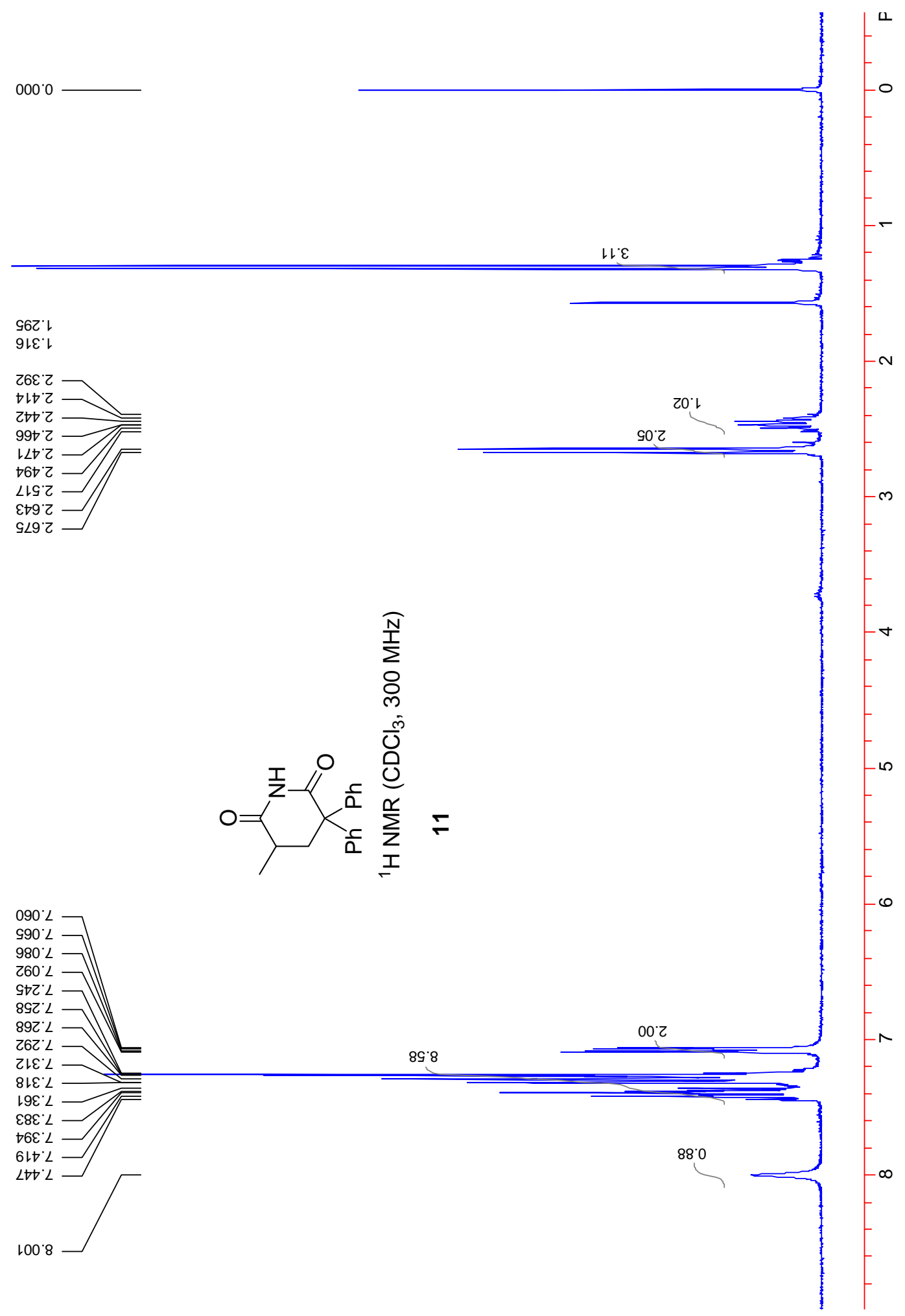




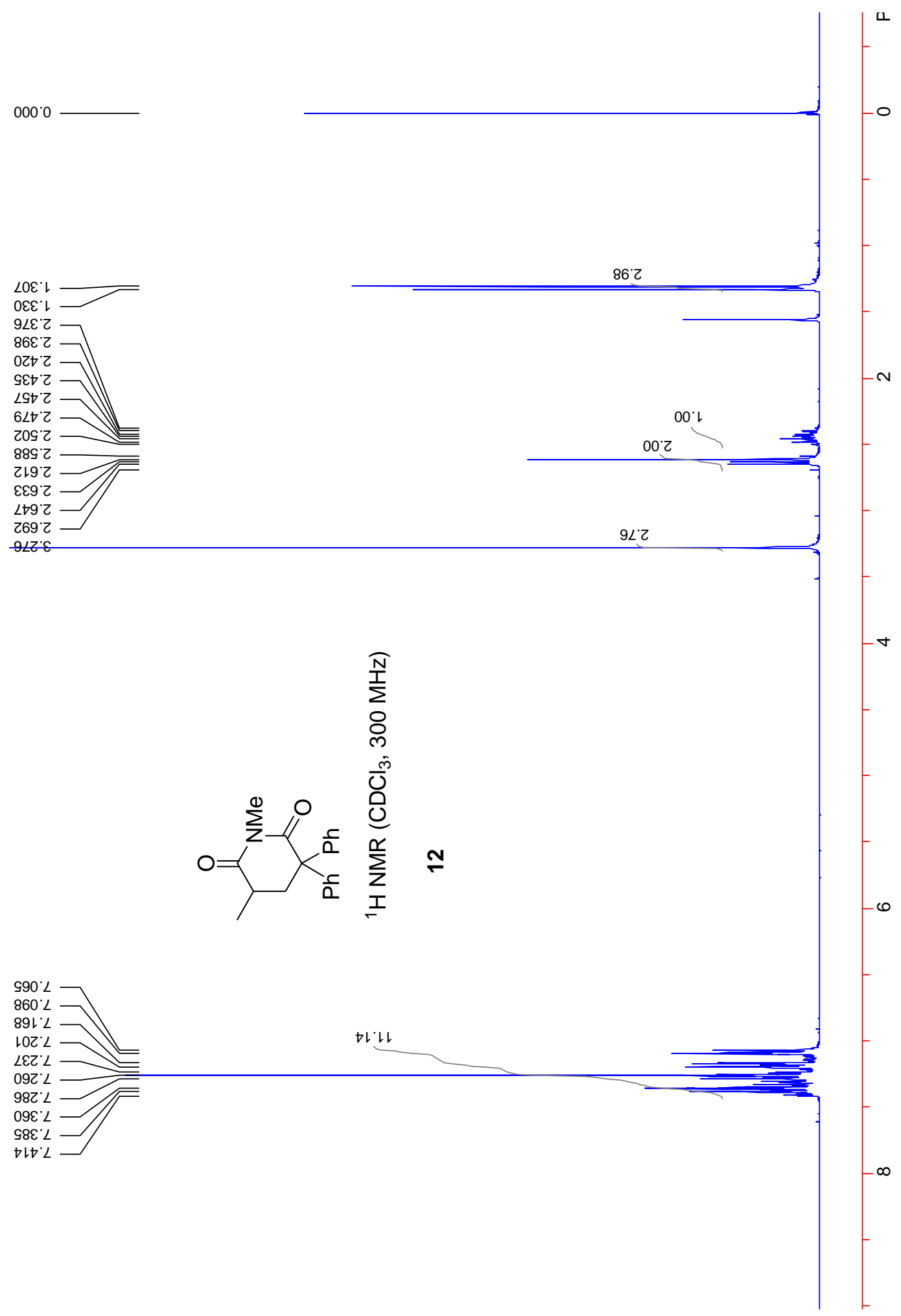


$\varepsilon 0 L^{\circ} 91$

$268^{\circ} \angle 2$

$8 \varepsilon 9^{\circ} \downarrow \varepsilon$

टह8' $8 \varepsilon$

$8 \& 8^{\circ} \angle 9$

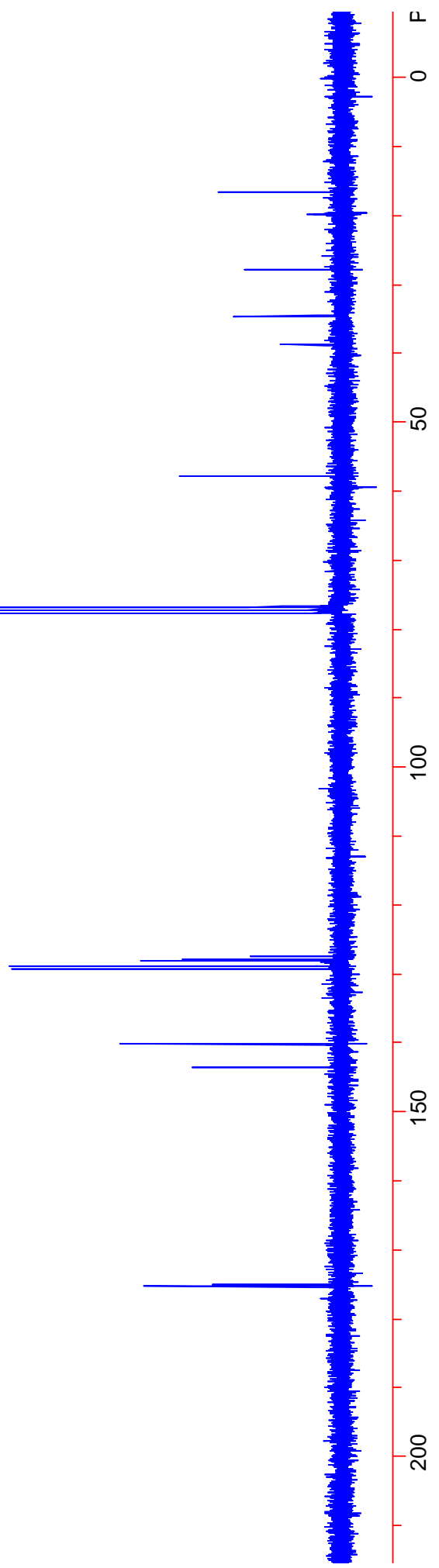

168.9

$9 t Z \angle L$

$\angle L 9 ' \angle L$

$\varepsilon S t \cdot L 21$

$908 \angle 2 t$

810.821

091.8Z1

ع08.8ZL

$\varepsilon \varepsilon Z^{\circ} 0 t$

8LG' $E$ เ

$186^{\circ} \rightarrow \angle L$

$69 \varepsilon^{\circ} \mathrm{SLt}$

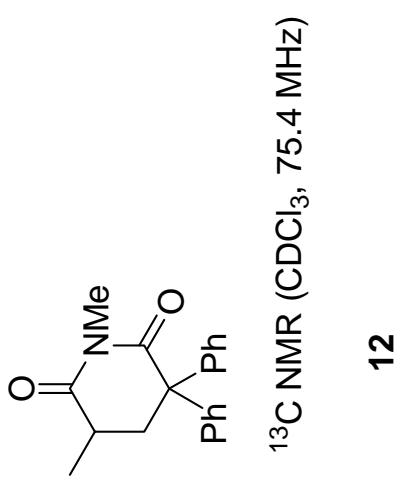




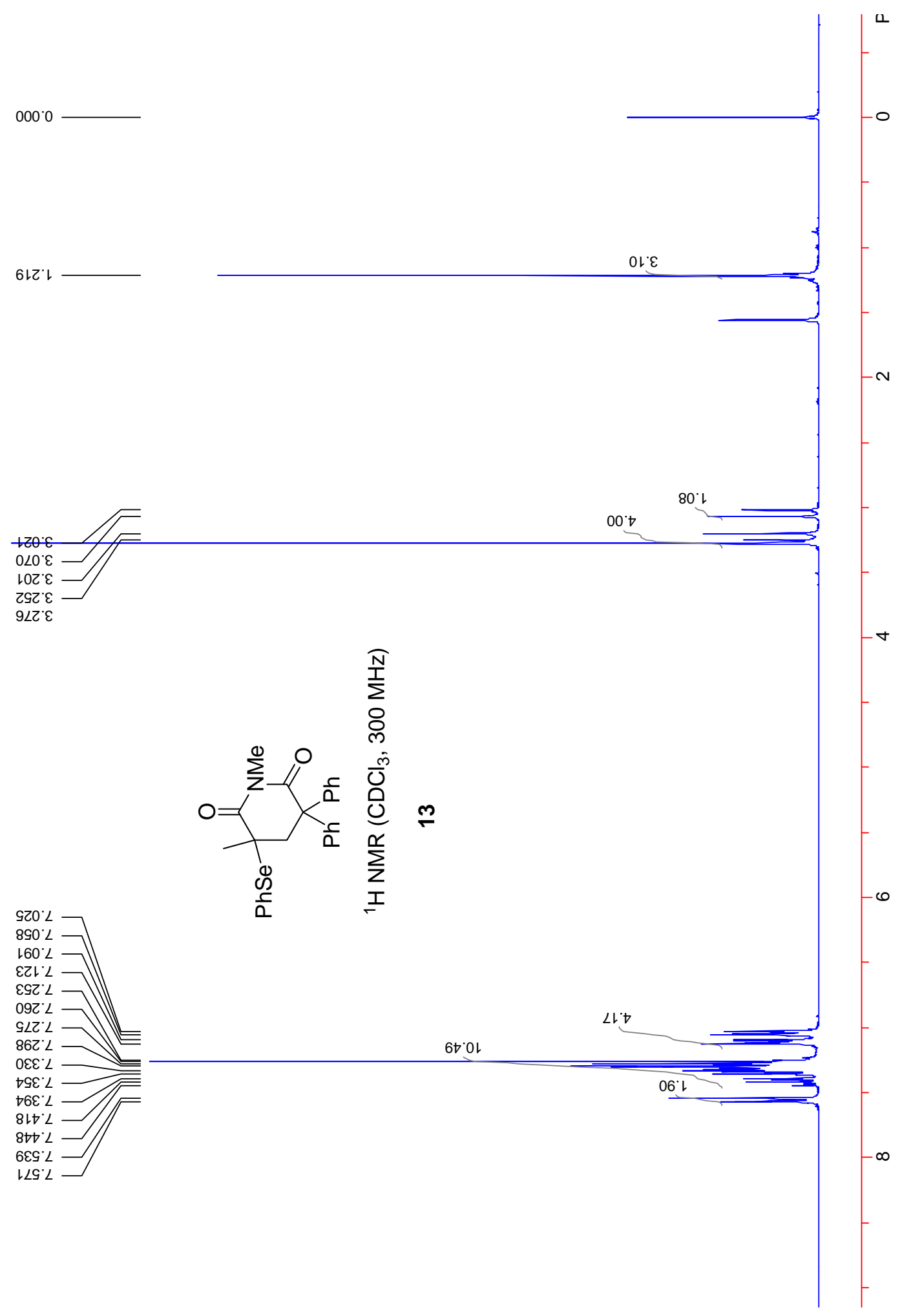


온

$9 \varepsilon+\cdot 92$

ZL8. 82

¿SS' $E t$

SLE'St

$\varepsilon 80^{\circ} \angle 9$

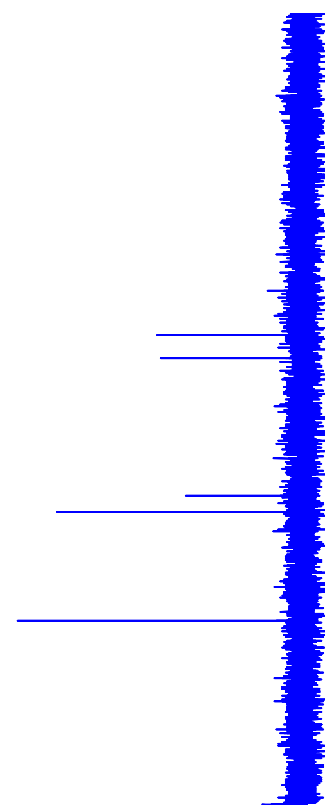

$692 \angle L$

$\varepsilon 89^{\circ} \angle L$

$\nabla Z 8 \cdot \angle 2$ †06 $\angle 21-$

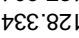

$\varepsilon 9 \varepsilon .82$ ।

$\varepsilon 98 \cdot 82$

$\angle \varepsilon+62 t$

991.6Zt

ट60 $0 \varepsilon 1$

†ย $8 \varepsilon$

206. Ltt

$91 \varepsilon$ ¿t। -
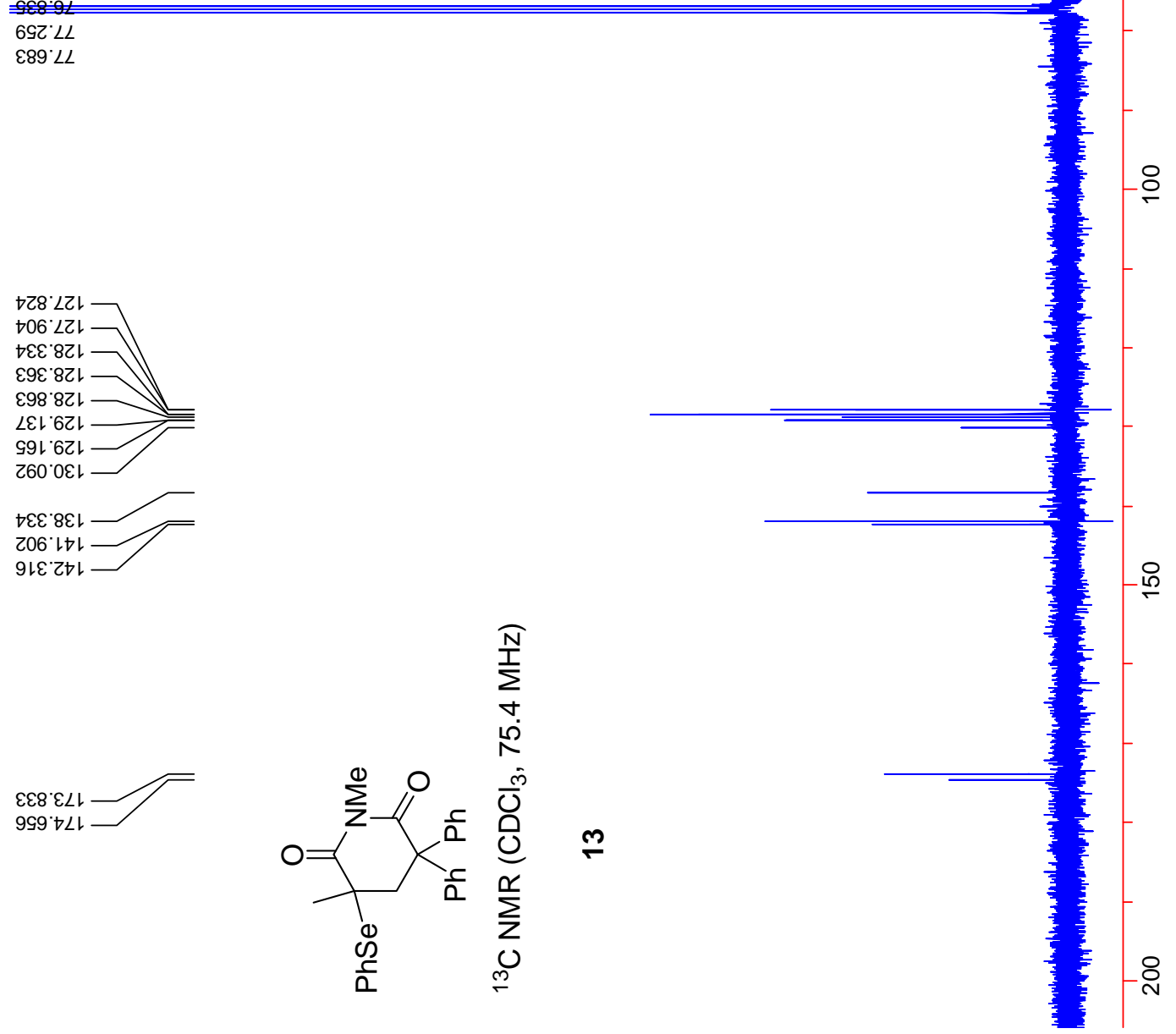


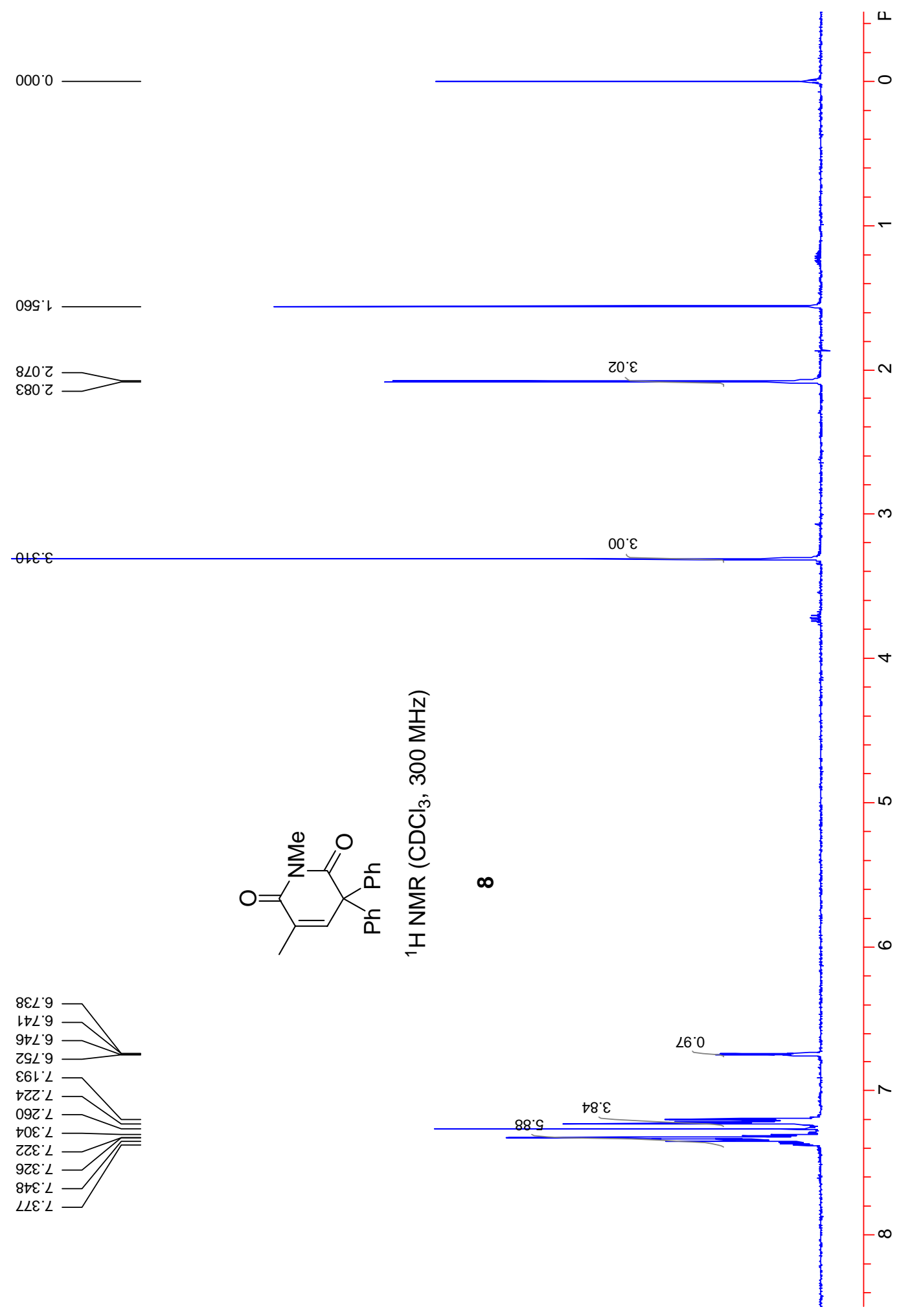


$\frac{1}{n}$

$\mid \angle 6^{\circ} 91$

$86 \cdot \div 2$

$968^{\circ} 69$

५\&8. $9 L$

997

$\varepsilon 89^{\circ}$ LL

9เ乙.92เ

टL0 $82 \mathrm{~L}$

$\angle \varepsilon 8^{\circ} 8 \mathrm{LL}$

†88 82 เ

乙EO०てヤト

109 \&†t $\longrightarrow$

¿†6.991 -

$80 L^{\circ} \forall \angle L$
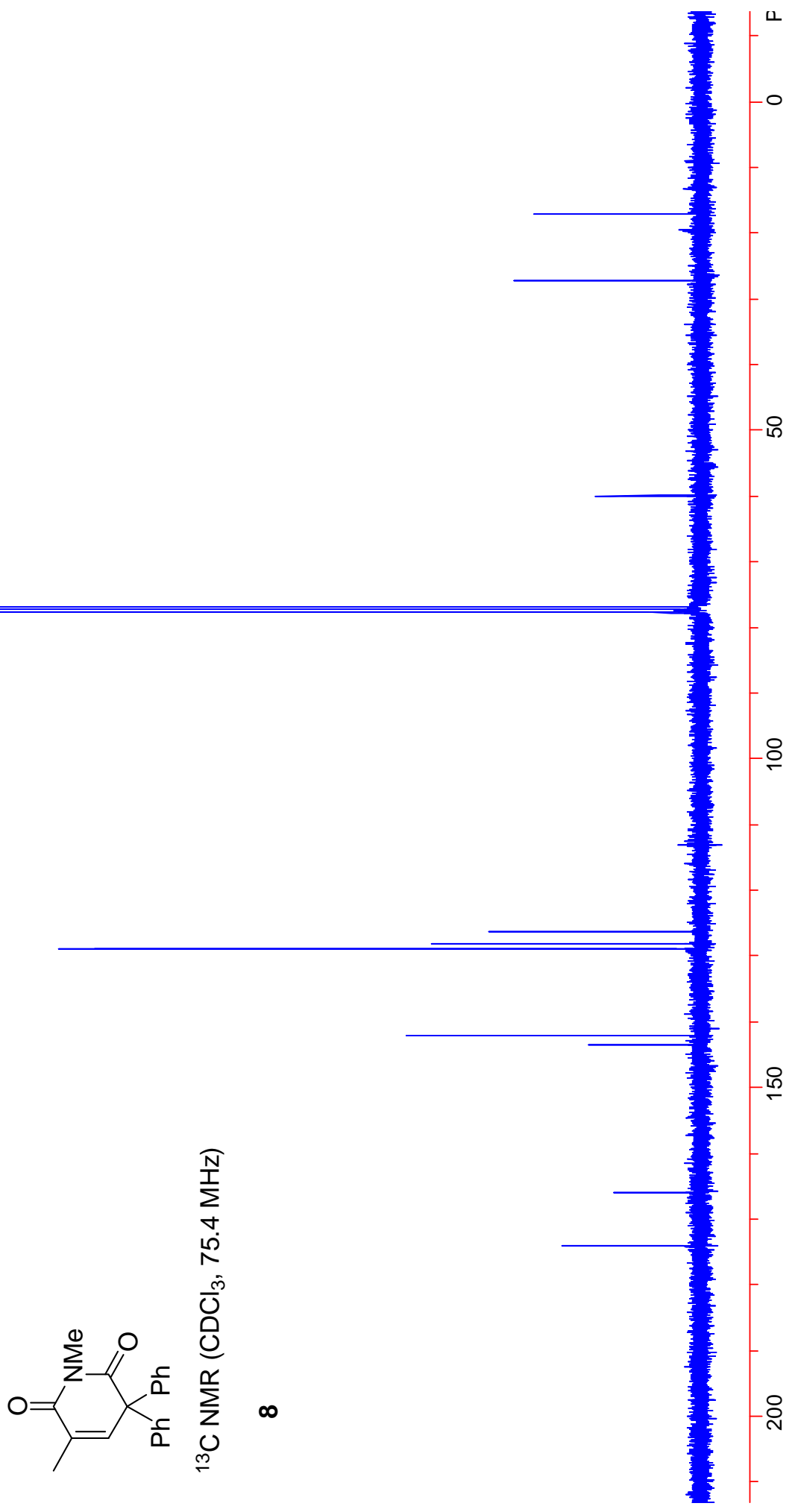
$\frac{m}{n}$

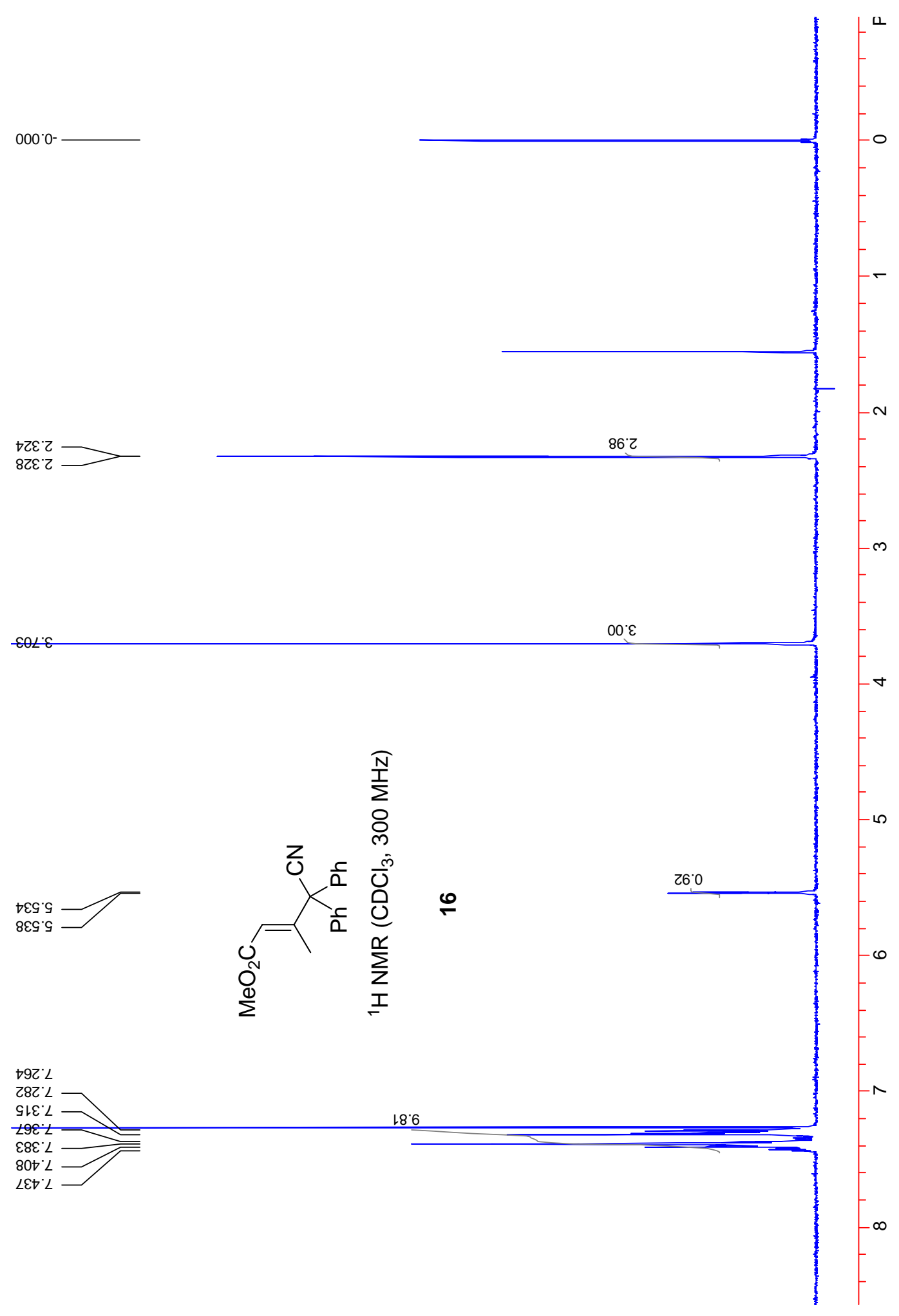


is

$899^{\circ} 81$

¿29. เ9

690.19

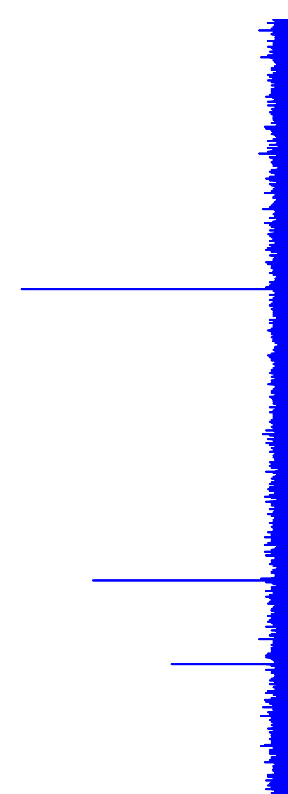

$\angle \measuredangle T^{\circ}$

$89 t^{\prime} \mathrm{ZZ}$

$9 \varepsilon 8.821$

$806^{\circ} 8 \mathrm{ZL}$

L6t'62t

Eเト LE
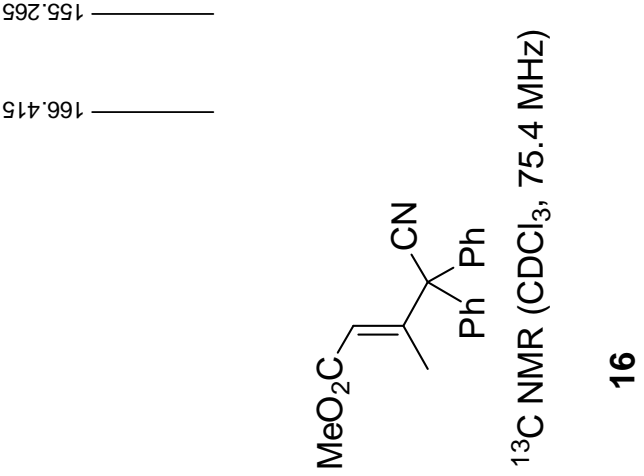
$\frac{n}{n}$
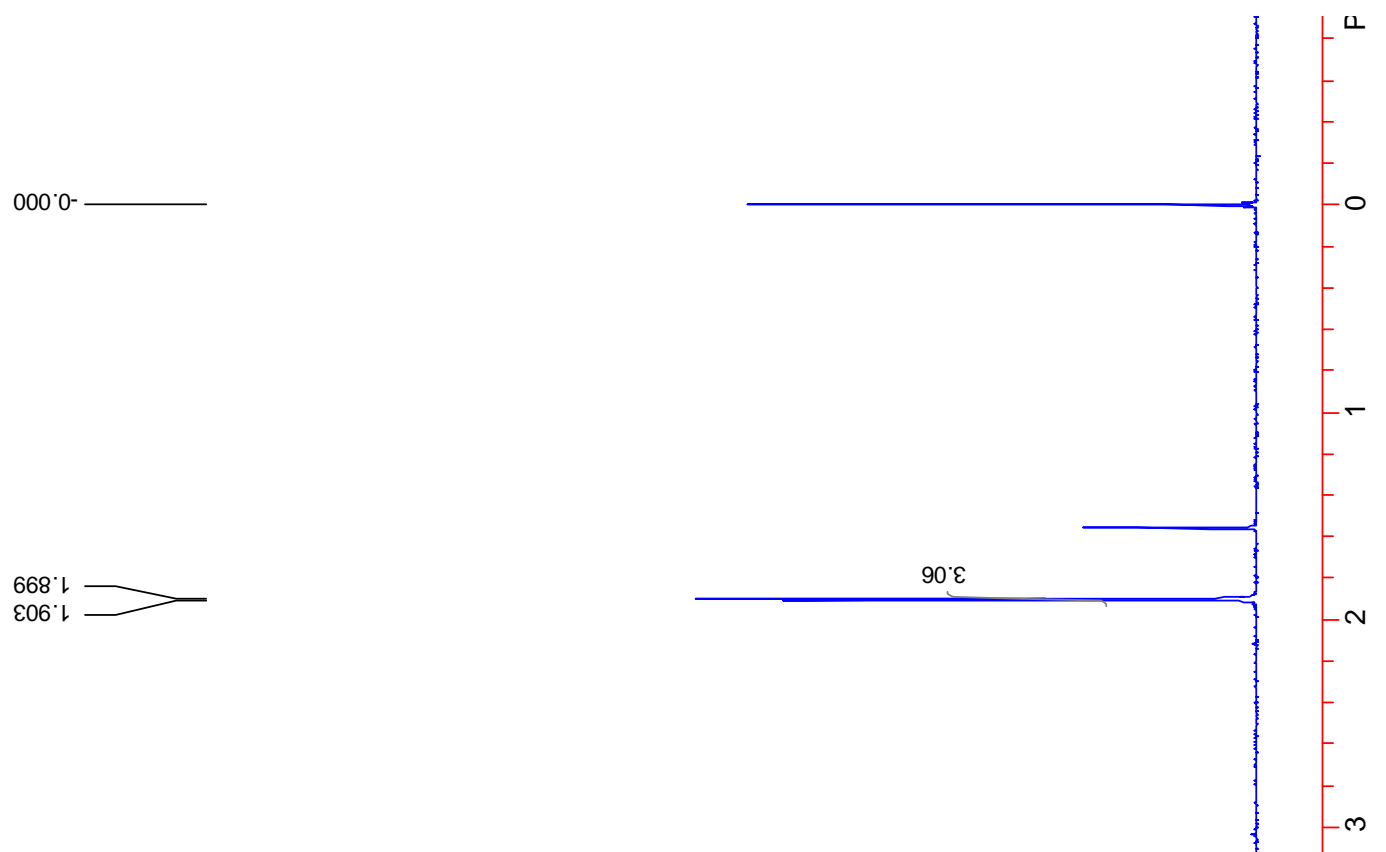

เ8乙 $\varepsilon$

$00^{\circ} \varepsilon$

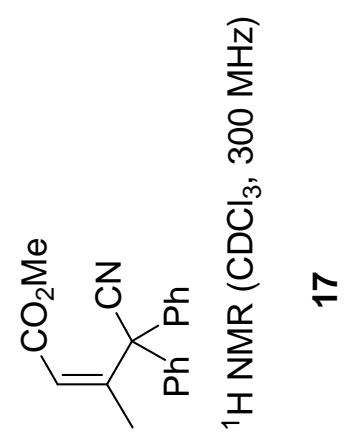

OSเ.9
ง เ $9 \square$

ट9ट L

LE $\varepsilon^{\circ} \angle$

L9E'L

S8E $L$

26.6

$96^{\circ} 0$ 
$\frac{0}{5}$

OZヤ ๑८

SZS'IS

†๑ะ $\angle S$

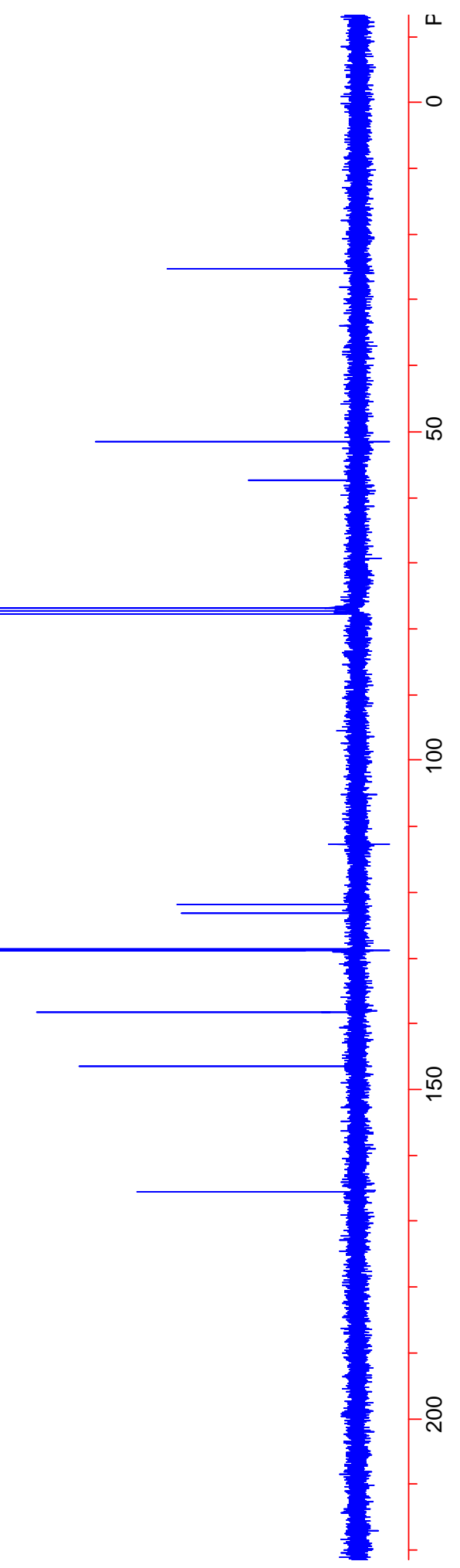

$\varepsilon \angle 8 \cdot L L \mid$

ट6เ' $\varepsilon ट t$

ธ๕५ $8 \mathrm{LL}$

St8. $8 \mathrm{Zt}$

$2988 \mathrm{CL}$

$86+\cdot 8 \varepsilon+$

$96 t \cdot 9 t r$

$6 \varepsilon$ เง9เ

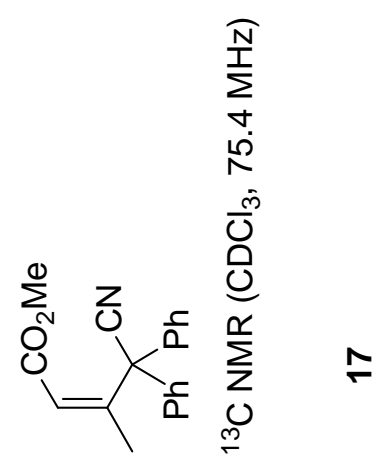


के

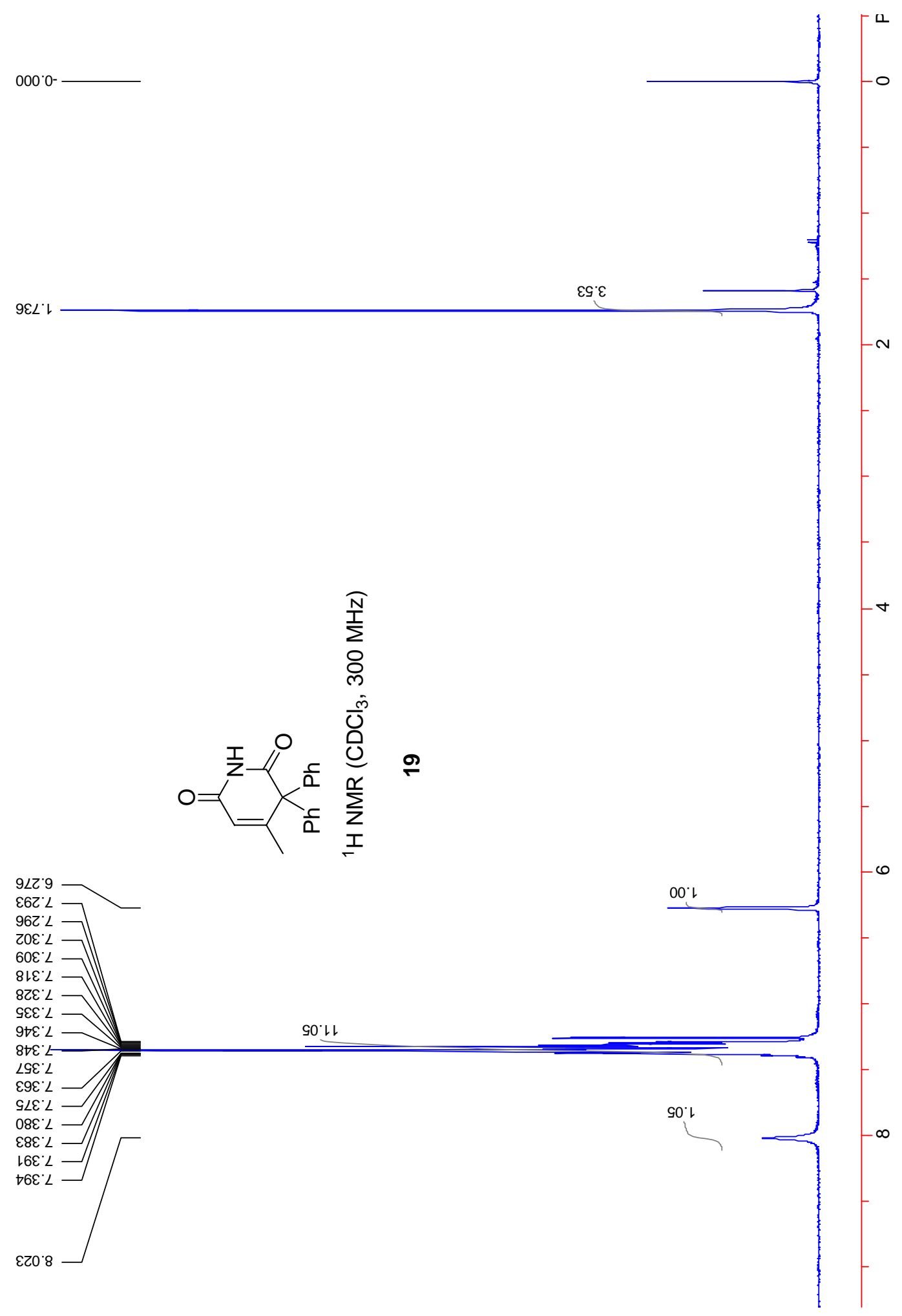


Z๐॰

५८८' $\overbrace{}$

†乙Ь $9 L$

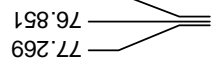

$692 \angle L$

$\angle t G \circ 6$

$866^{\circ} \angle 2 L$

$\frac{\angle 9 \varepsilon .82 t}{00 \varepsilon^{\circ} 62 t}$

$\angle 89^{\circ} 8 \varepsilon+$

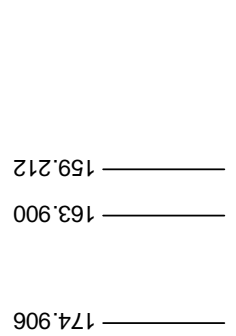

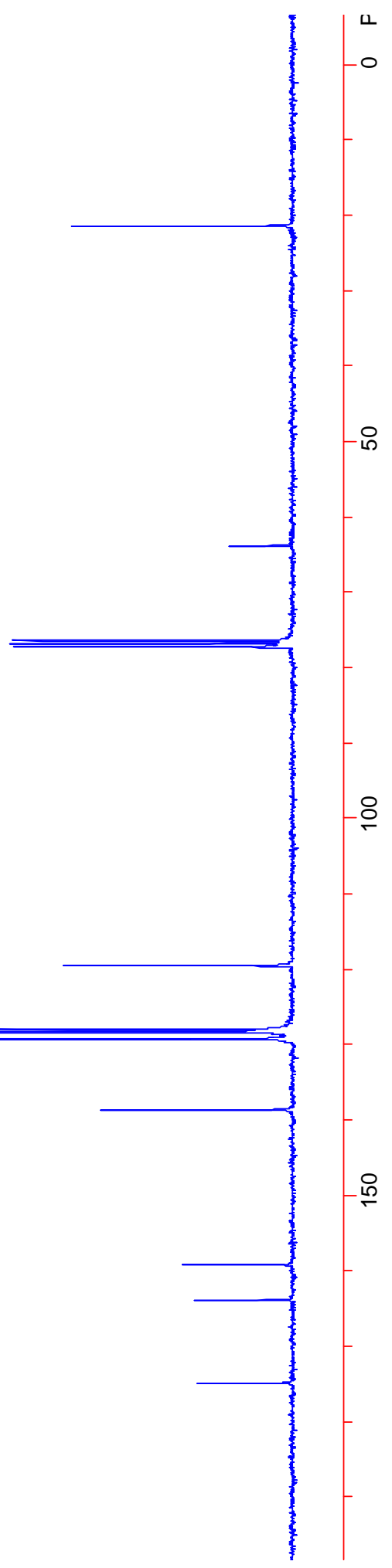


$\frac{a}{n}$

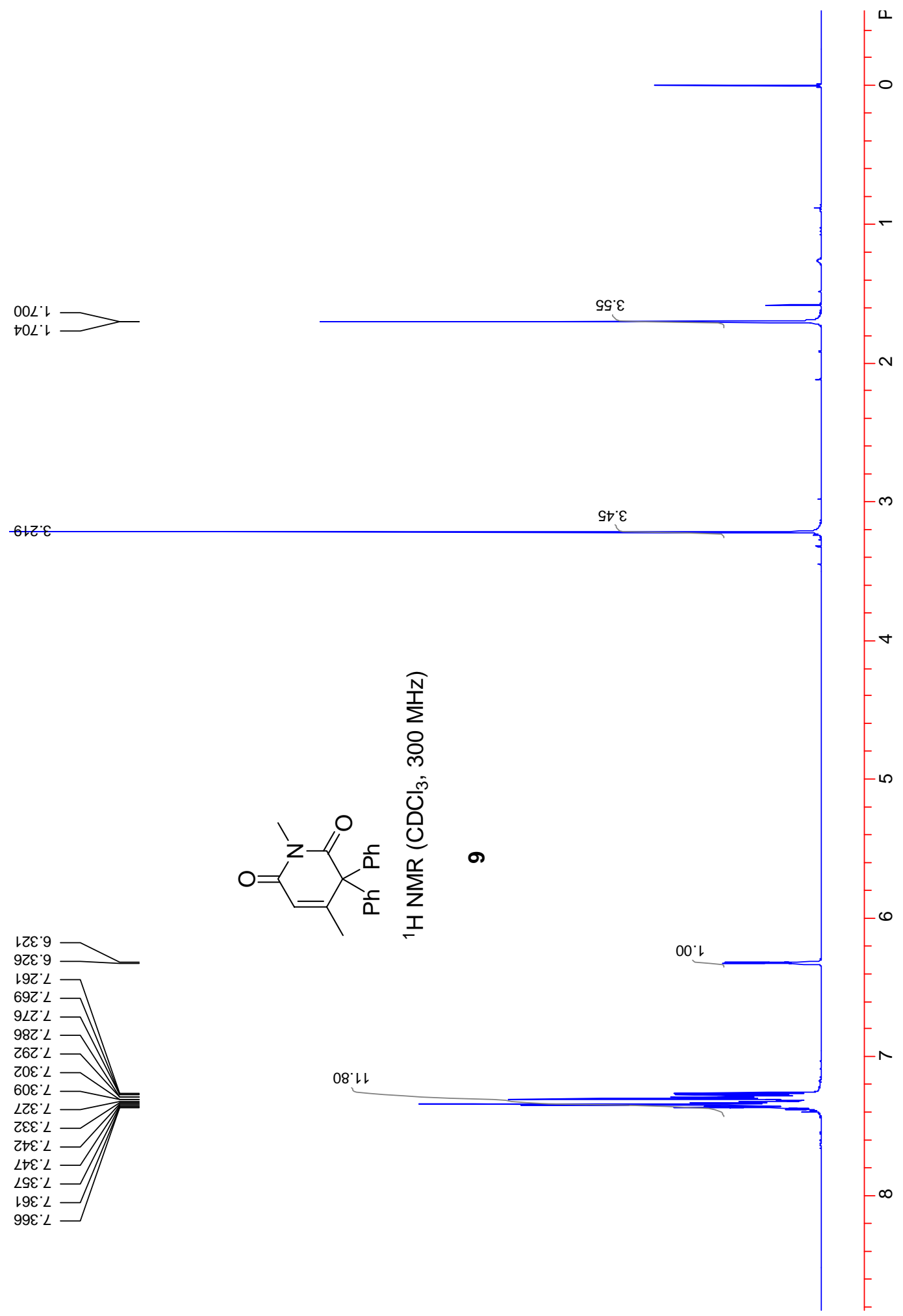


ชิ

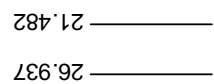

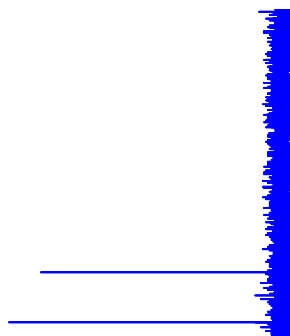

Etl.t9

$\angle E 88^{\circ} 9 L$

$\frac{ \pm 2 \% \angle L}{889 \angle L}$

†0†・0Zト

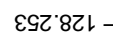

$90 L^{\circ} 8 \mathrm{ZL}$

$8 \angle 9^{\circ} 62 \mathrm{~L}$

$\varepsilon 8 L^{\prime} 6 \varepsilon$

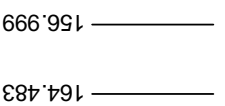

$698^{\circ} \varsigma \angle L$

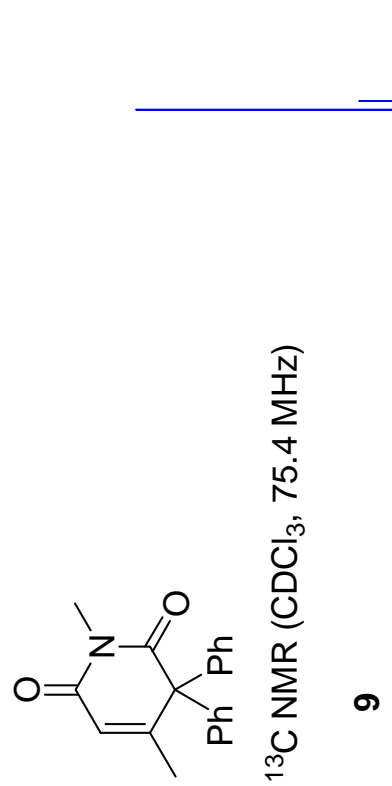




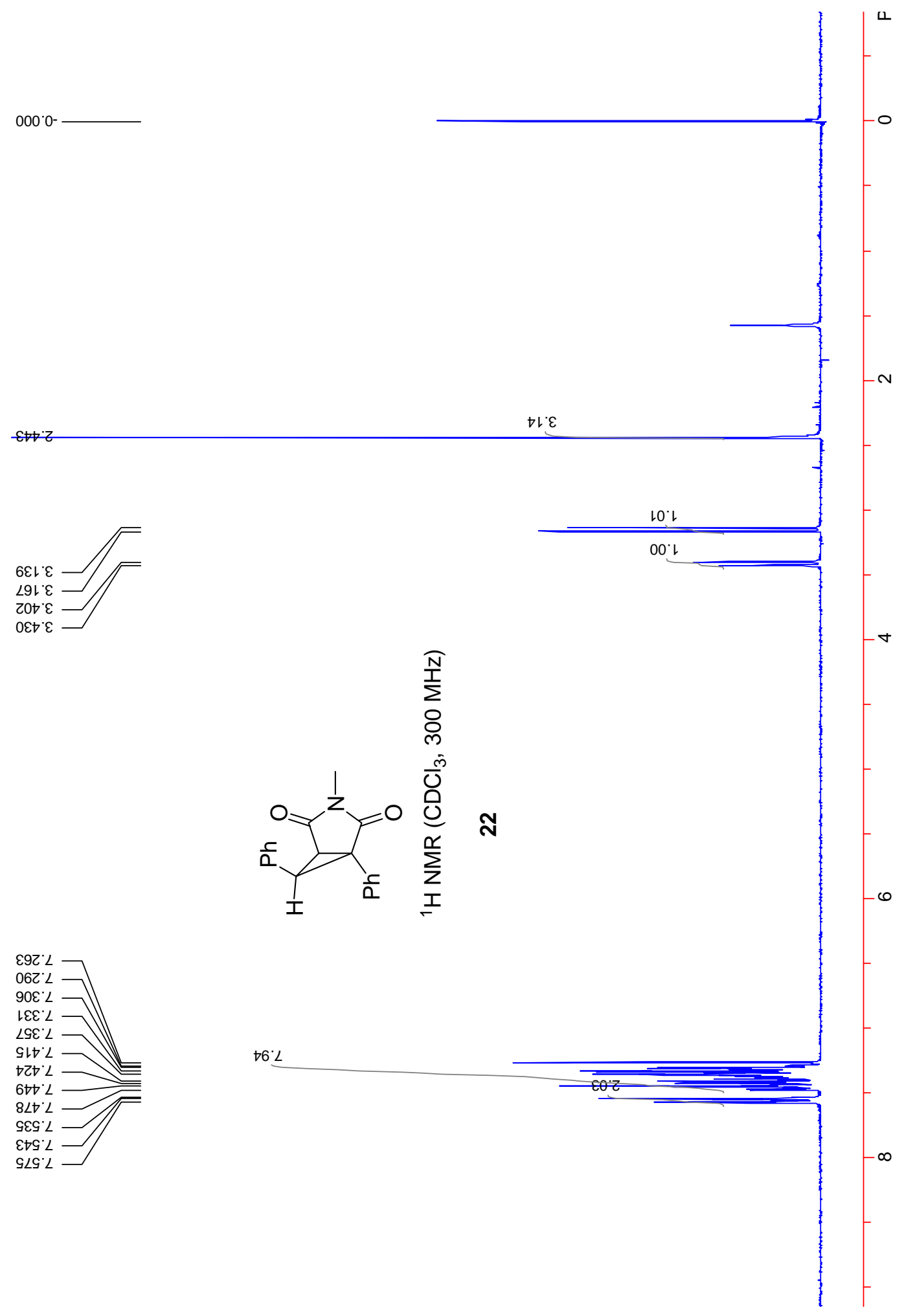


สี่

$8^{\circ} 6^{\circ} \varepsilon Z$

6己เ`てะ
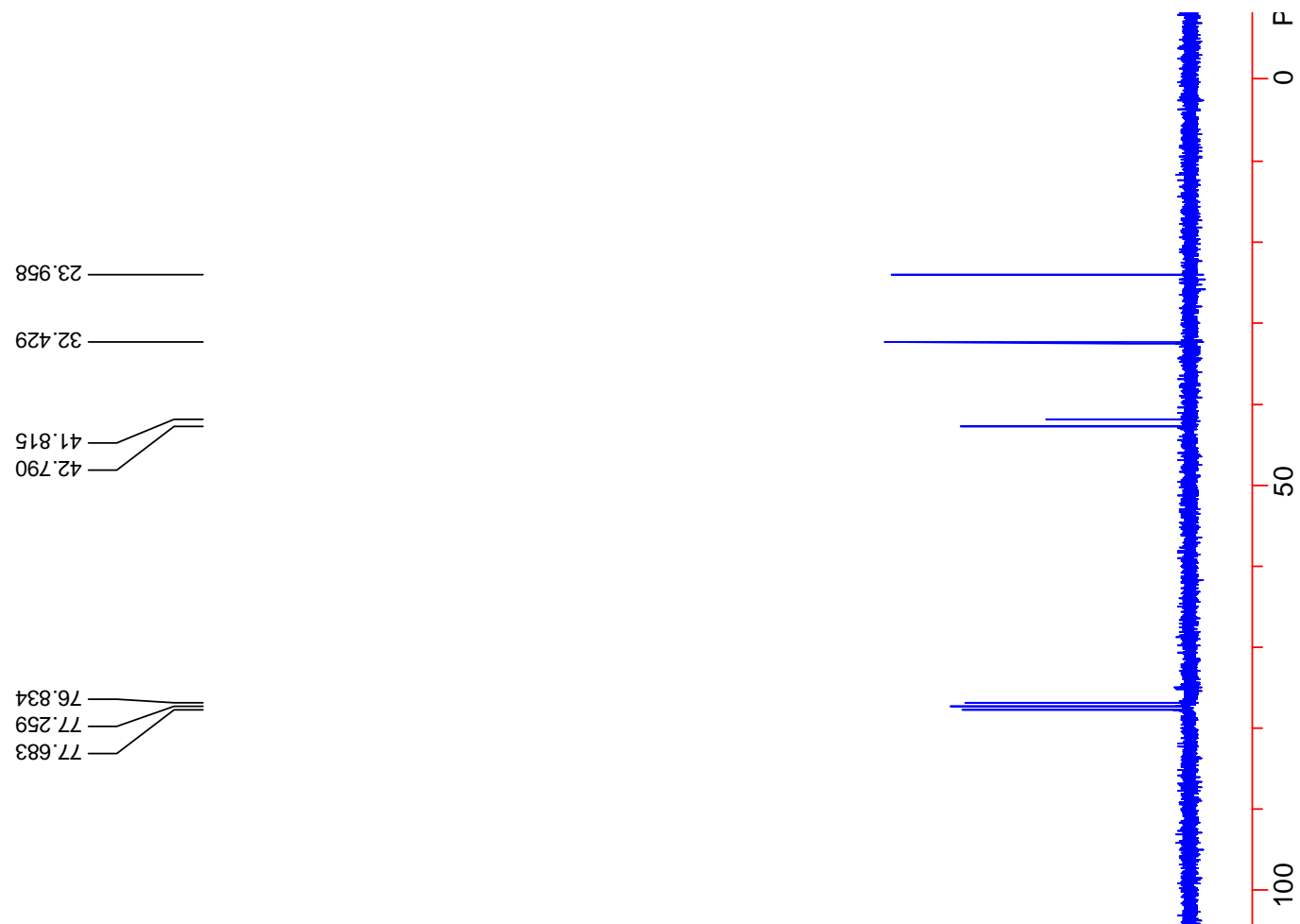

†ย८.9L

$692 ' \angle L$

$\varepsilon 89^{\circ} \angle L$

89t' 821

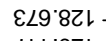

$\angle L+62$

tSI. $62 \mathrm{~L}$

$9 \angle 1 \cdot 621$

$0 \angle Z 6 Z 1$

898' $2 \varepsilon 1$

066 ' $2 \varepsilon$
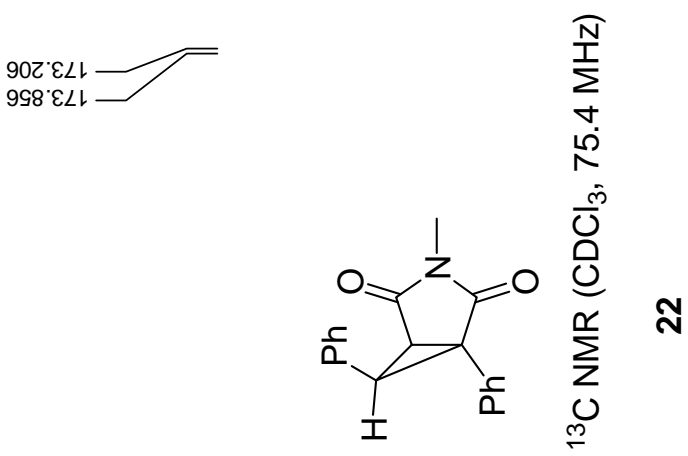

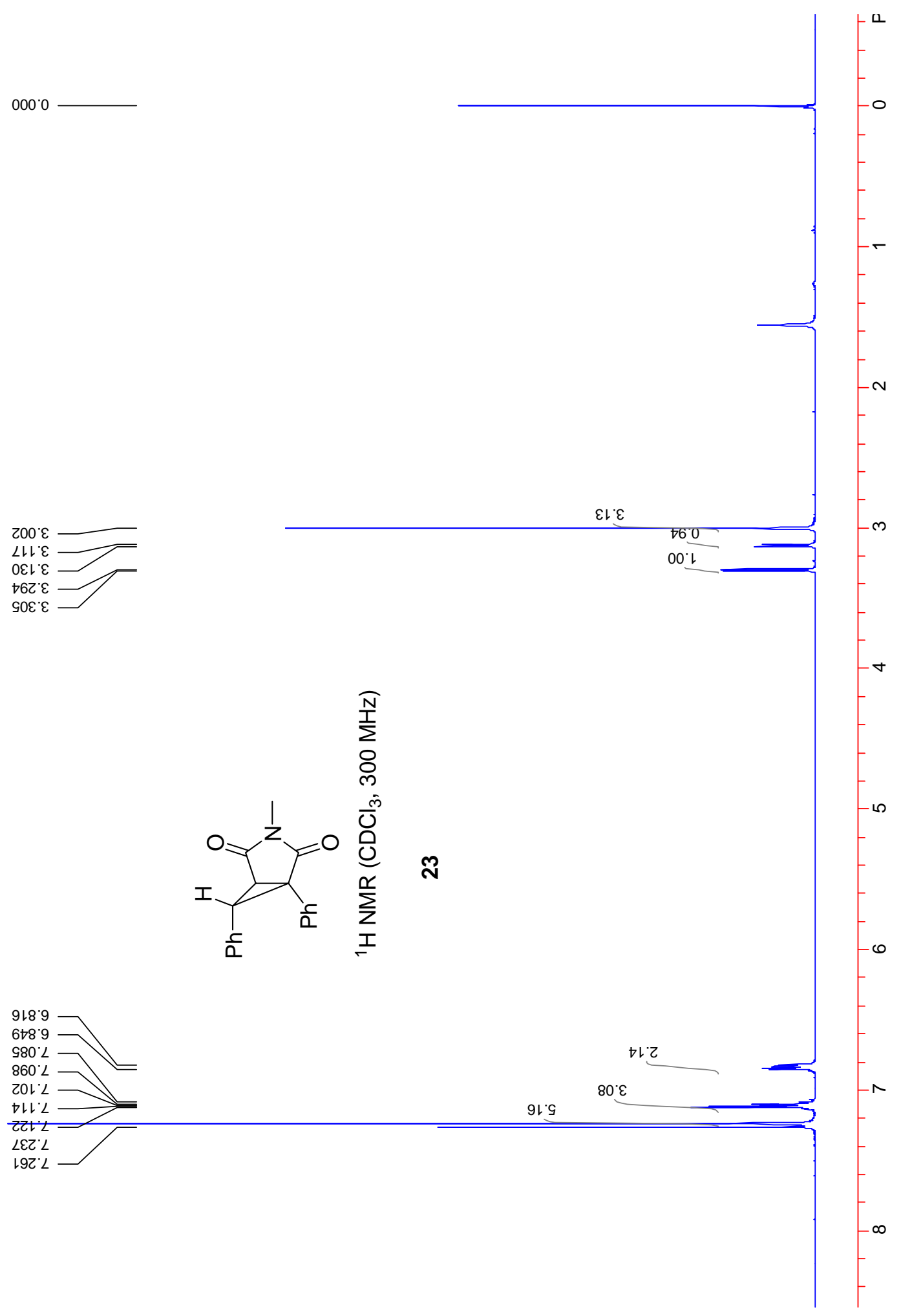


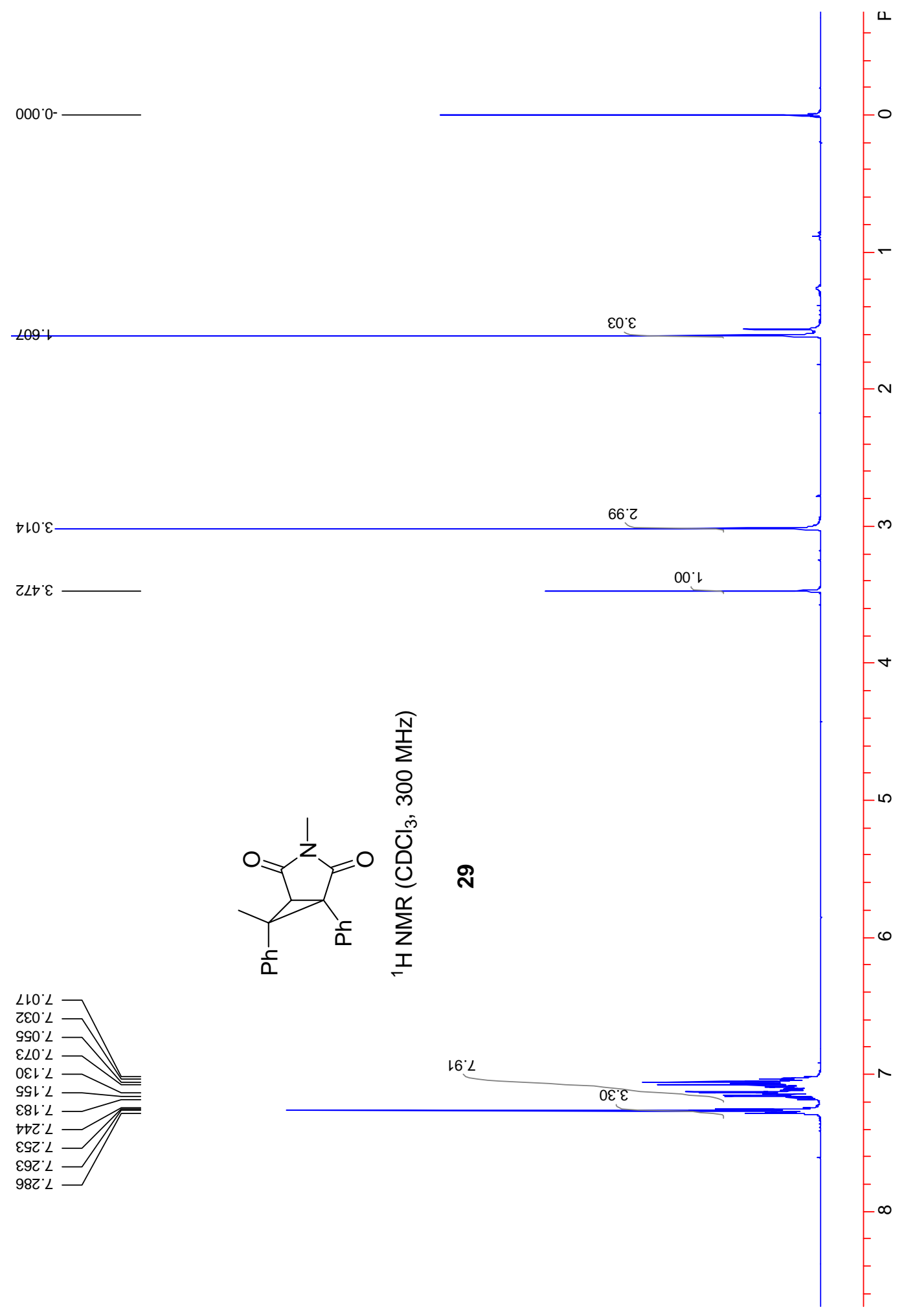


$\varepsilon 06.6 \mathrm{t}$

$6+5{ }^{\circ} t z$

$060^{\circ} \downarrow \varepsilon$

$\varepsilon+L ' S t$

$6 \angle 6{ }^{\circ} 8 t$

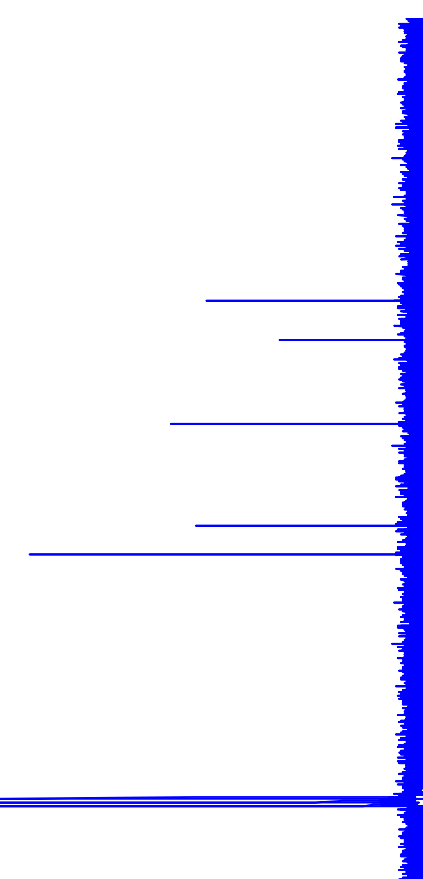

ㄴ

†ะ० $9 L$

892

$289^{\circ} L L$

ESt $\angle 2 L$

$6 t \angle \angle Z L$

$8 \mathrm{~L} 8 \mathrm{~L}$

$\angle 8 t^{\circ} 8 \mathrm{ZL}$

$0+\angle ' B Z 1$

เEเ 621

५Z9.0ह।

$6 \nabla L ' 6 \varepsilon$

$\supset ट 8^{\circ} \varepsilon \angle L$

ट9ナ" $\bullet \angle L$

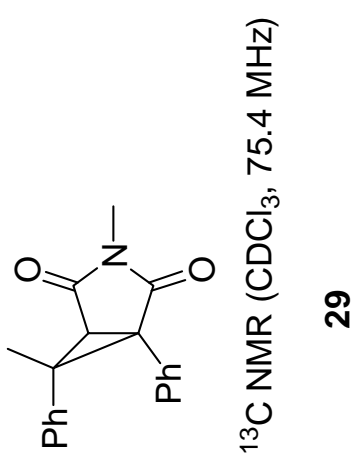




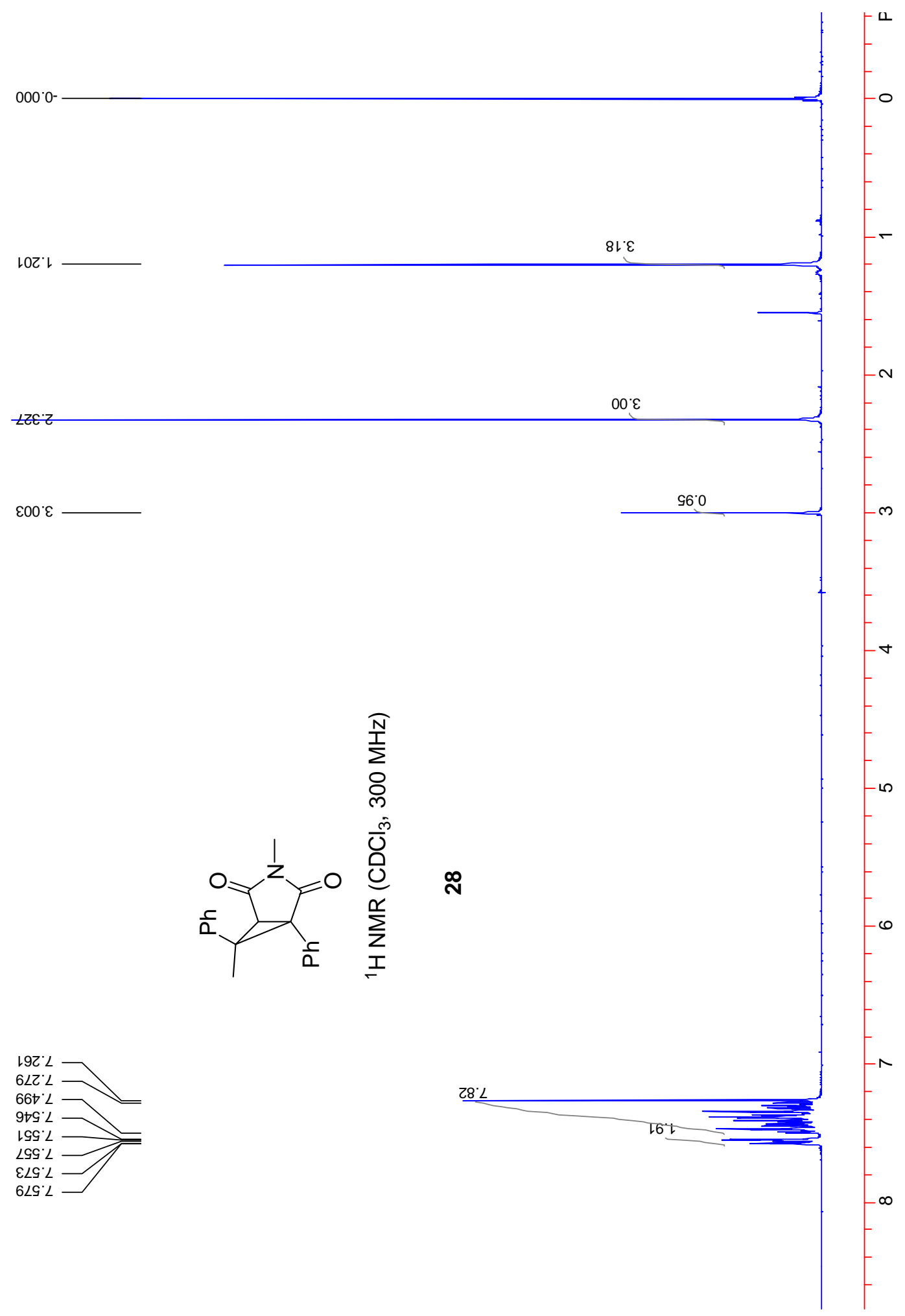


초

เร6. $๕ 2$

StD' $\mathrm{CZ}$

$\tau t \angle \cdot 9 \varepsilon-$

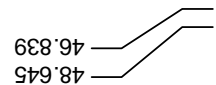

เtt. 821

$20 t \cdot 82$

दL9 82

$866^{\circ} 82 \mathrm{~L}$

8हट 62 .

$\varepsilon \varepsilon \varepsilon \cdot 0 \varepsilon$

乙St०0ع।

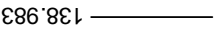

$\varepsilon \nabla 8^{\circ} \varepsilon \angle 1+$

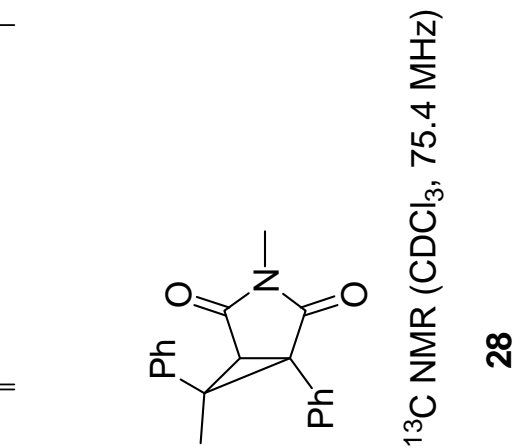

$\stackrel{\infty}{\sim}$

$06 \varepsilon^{\circ} \triangleright \angle L$

m

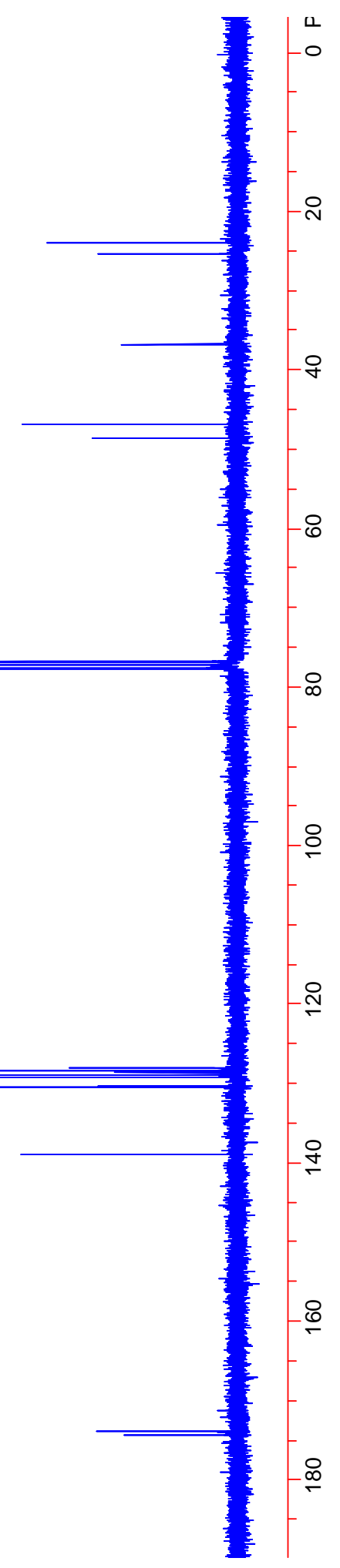


$\stackrel{\infty}{\mathscr{N}}$

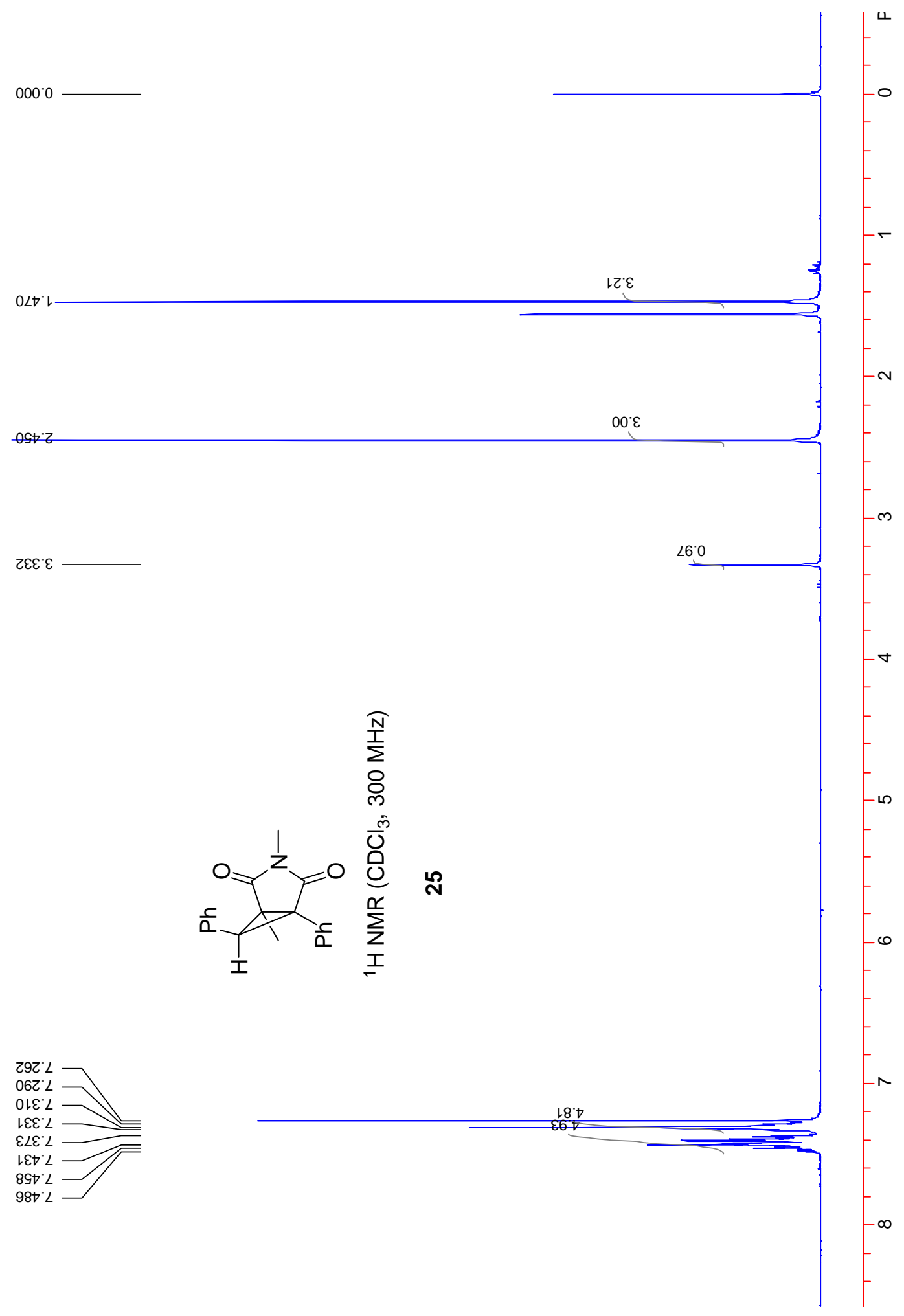


๙ิ

๑60`て -

sto $t z$

$\varepsilon$ เ ' $8 \varepsilon$

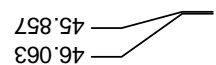

$828.9 L$

$8+Z Z L L$

$\mathrm{S} \angle 9^{\circ} \angle L$

$682 \cdot 8 \mathrm{~L}$

$898^{\prime} 8 \mathrm{ZL}$

$890^{\circ} 6 \mathrm{Zl}$

SLO 621

ยहट 62 เ

$880^{\circ} 0 \varepsilon 1$

$8 \angle 9 \cdot|\varepsilon|$

$6 \pitchfork 9^{\circ} \varepsilon \varepsilon เ$

$s \angle L D L t \longrightarrow$

IIG $S \angle L$
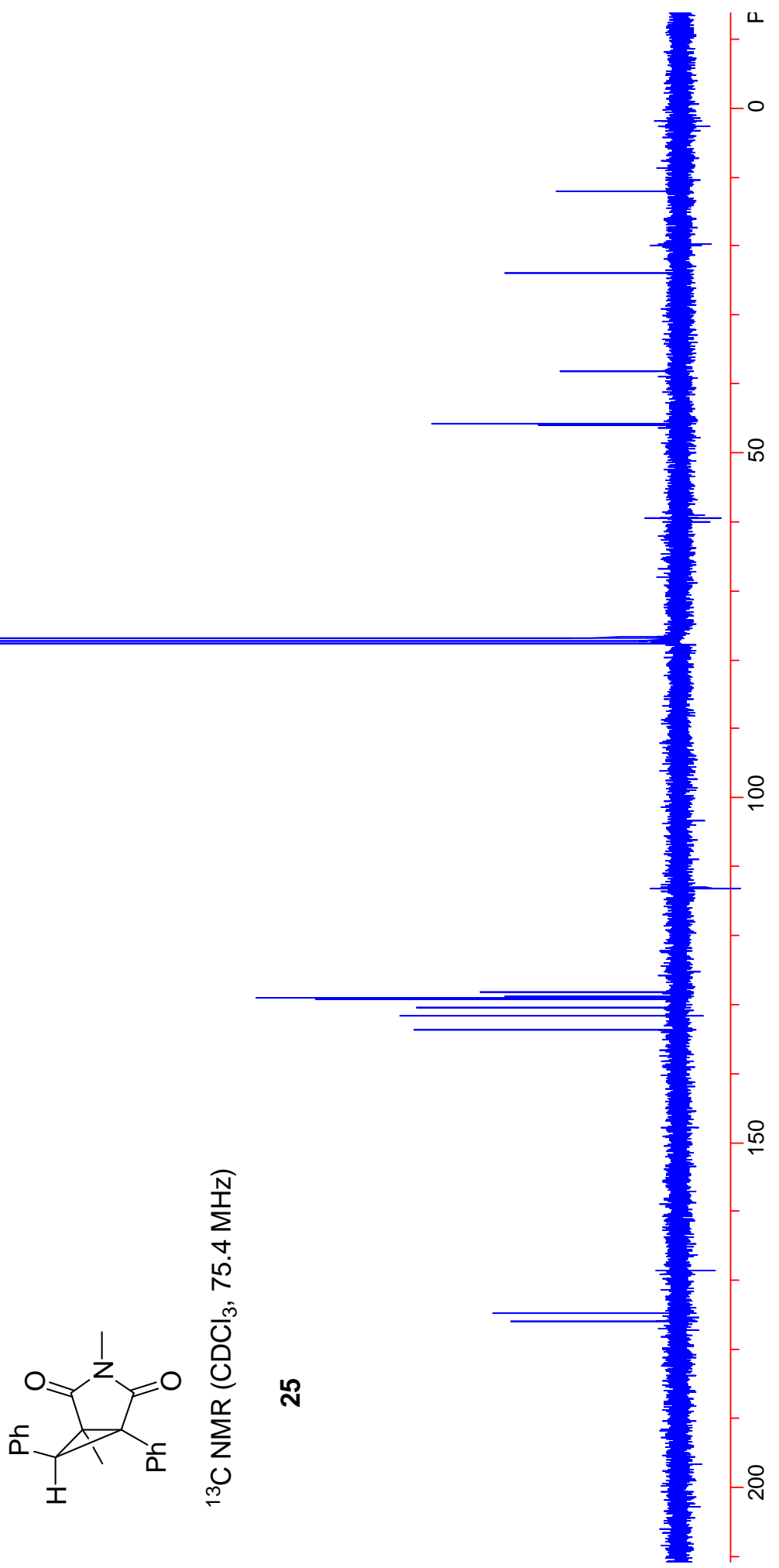
in

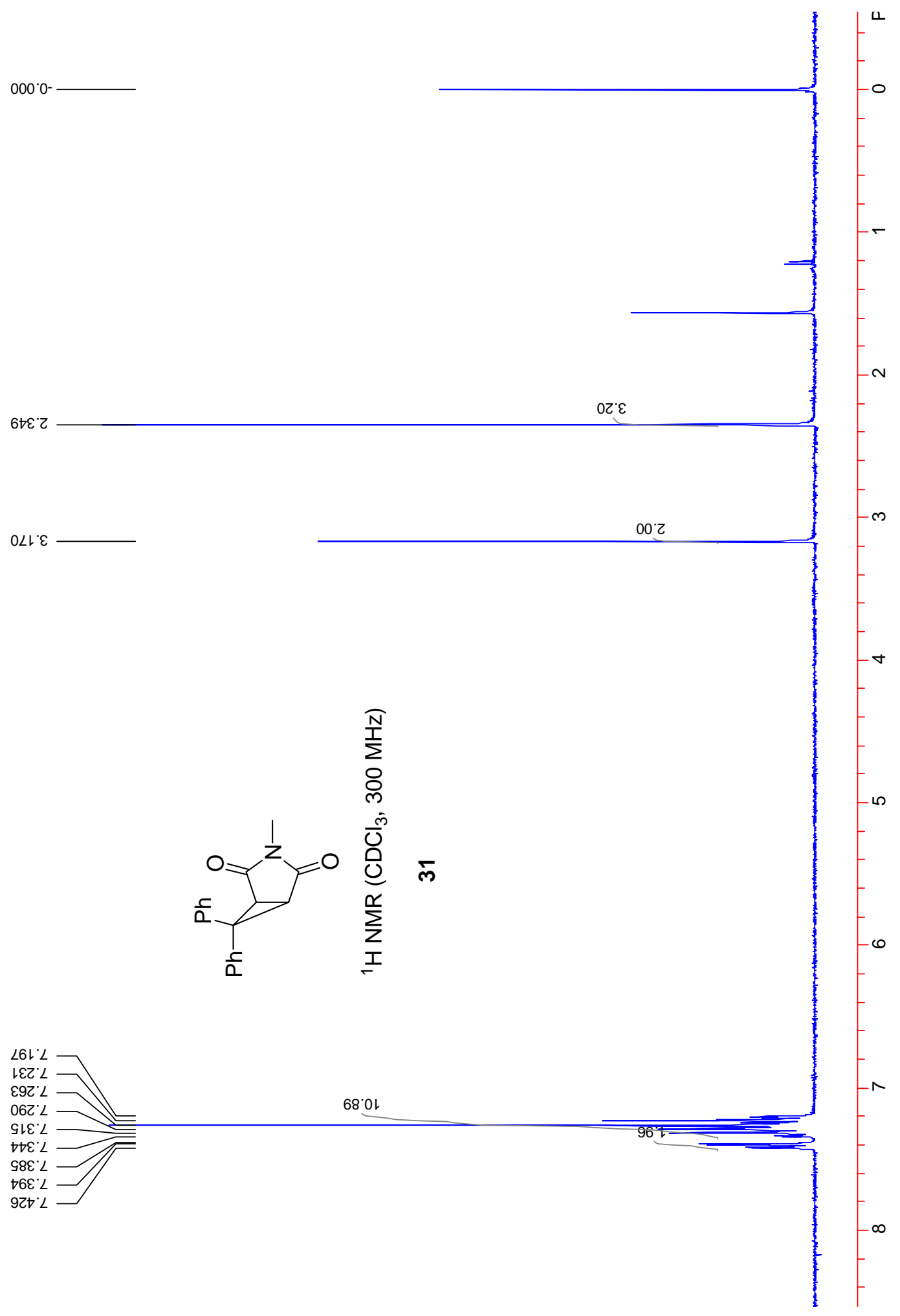


$\angle 88^{\circ} \mathcal{E}$

เトเ๋ย

sStos

$9 \varepsilon+\angle Z$

006.221

ESt $8 \mathrm{ZL}$

$\angle 0 \mathrm{~L}^{\circ} \mathrm{CZ}$

SOع.6己l

9๕9'6己।

५6ع.9ع।

$\angle 6 \varepsilon$ เトト

$6+Z \cdot L L$

$899^{\circ} \angle L$

$\dashv เ \varepsilon \cdot \varepsilon<t$
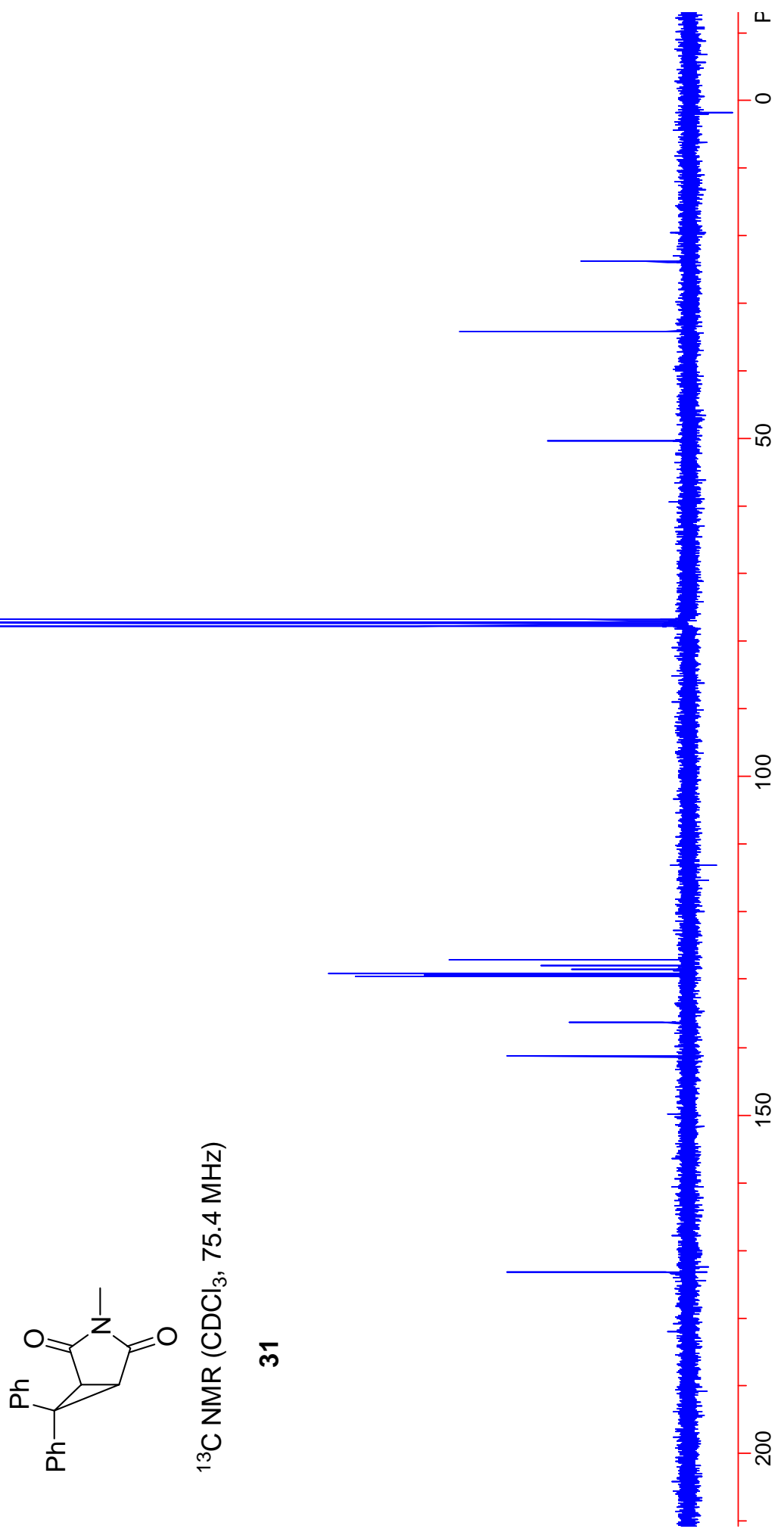
है

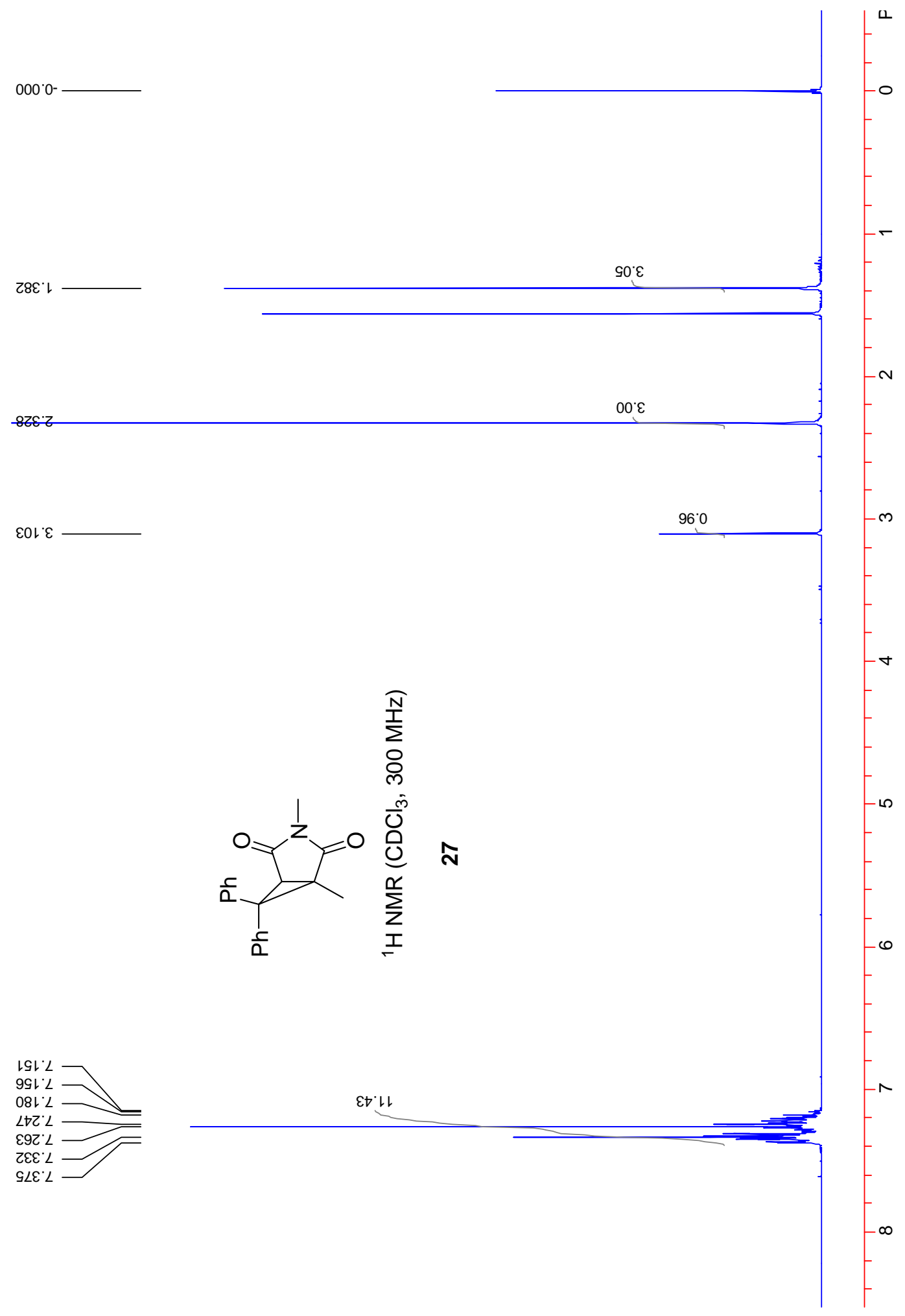


@

こยL’เレー

๕८8'๕乙-

$\varepsilon เ 8^{\circ} \mathcal{- 1}$

乙Z६. $\angle \varepsilon$

$89 \overbrace{}^{\circ} \downarrow 9$

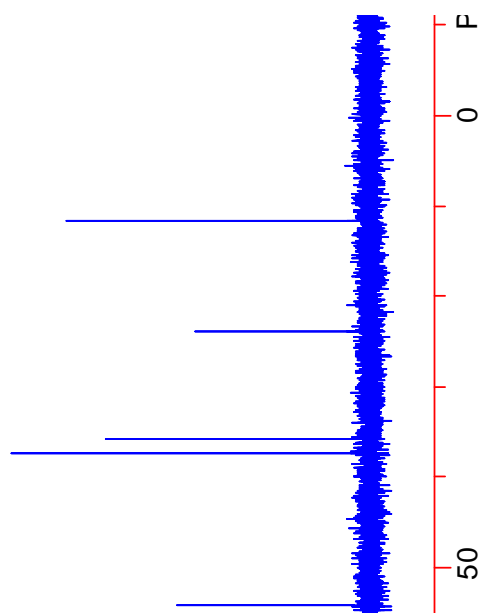

928.92

SGL LL

t89 LL

$268 . \angle 2$ $6 \angle 6 \circ 2 L$

†99.82t

ع06. 82 L

나다

$98 \mathrm{I}^{\circ} 6 \mathrm{~L} \mathrm{~L}$

† $\triangle L^{\circ} 8 \varepsilon \downarrow$

५68 $8 \varepsilon 1$

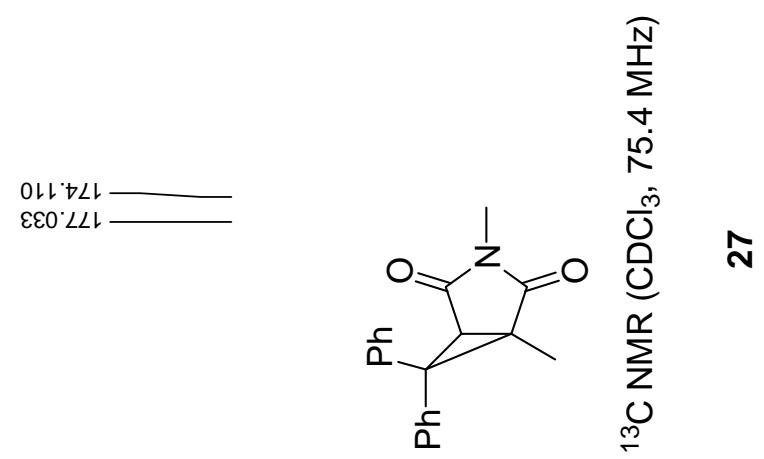

$N$ 


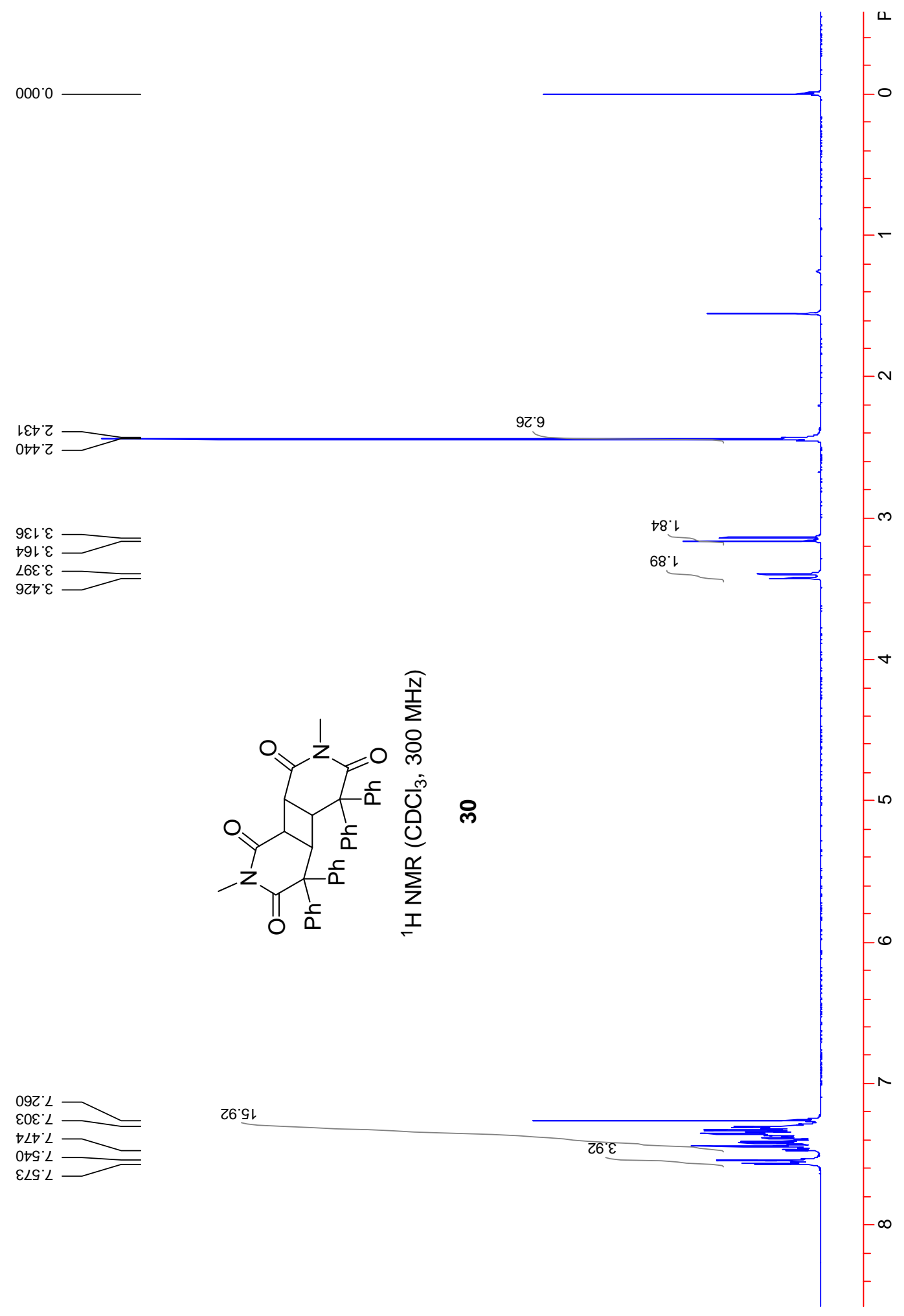


$\aleph_{\infty}^{\infty}$

$\angle \downarrow \mathcal{G}^{\circ} \varepsilon Z$

$\varepsilon 86^{\circ}\llcorner\varepsilon-$

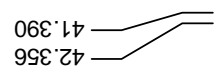

$86 \varepsilon^{\circ} 9 L$

$6288^{\circ} 9 L \longrightarrow \longrightarrow$
$99 C^{\circ} \angle L \longrightarrow$

$0+0.8 \mathrm{Lt}$ $\angle L Z \cdot 821$

90L' $8 \mathrm{LL}$

$t \mathrm{t} L \cdot 8 \mathrm{~L} \cdot$

$6 \varepsilon 8.8 \mathrm{LI}$

Lเt'ZEL

SLG'ZEL —

$+18^{\circ} 2 \angle L$

$+9 t^{\circ} \varepsilon \angle 1-$

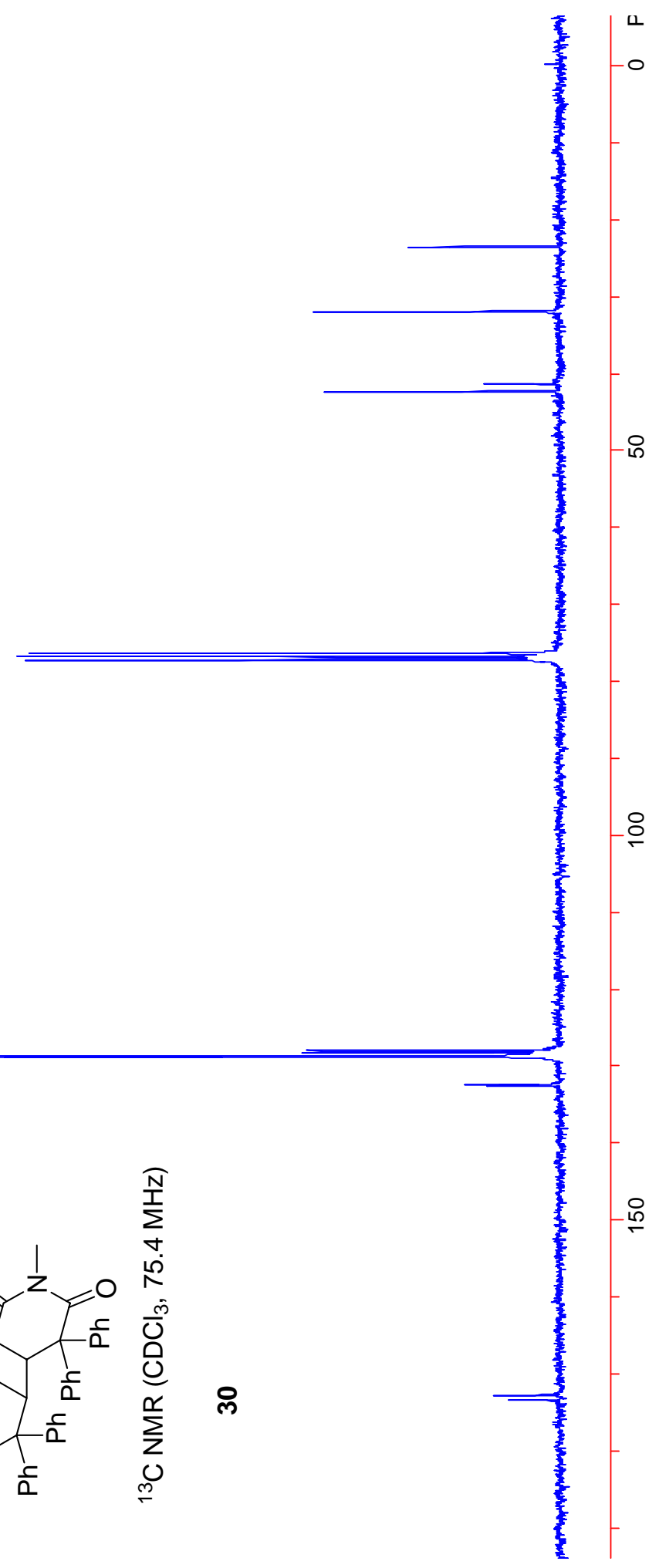


In parallel with our experimental efforts, and in order to better understand the reaction course, we turned to ab initio computations. $\operatorname{CASSCF}(8,8) / 6-31 \mathrm{G}(\mathrm{d})$ computations and NBO analyses on compound $\mathbf{2}$ showed that the lowest Franck-Condon triplet excited state formed (i.e. with S0 geometry) is $\pi-\pi^{*}$. Such triplets are known to result in $[2+2]$ additions ${ }^{5}$. The second excited state and relatively close in energy is an $n-\pi^{*}$ triplet which is usually responsible for aryl migration.

Interestingly, in another set of computations, with $\mathrm{T}_{1}$ optimized geometry, $\mathrm{T}_{2}$ exhibited two roots, both with odd-electron densities at carbon-5. Note Table 1 . The matching odd- electron density was at oxygen 7 , either in the $\pi$-system or in the $p_{y}$ orbital, depending the state. 

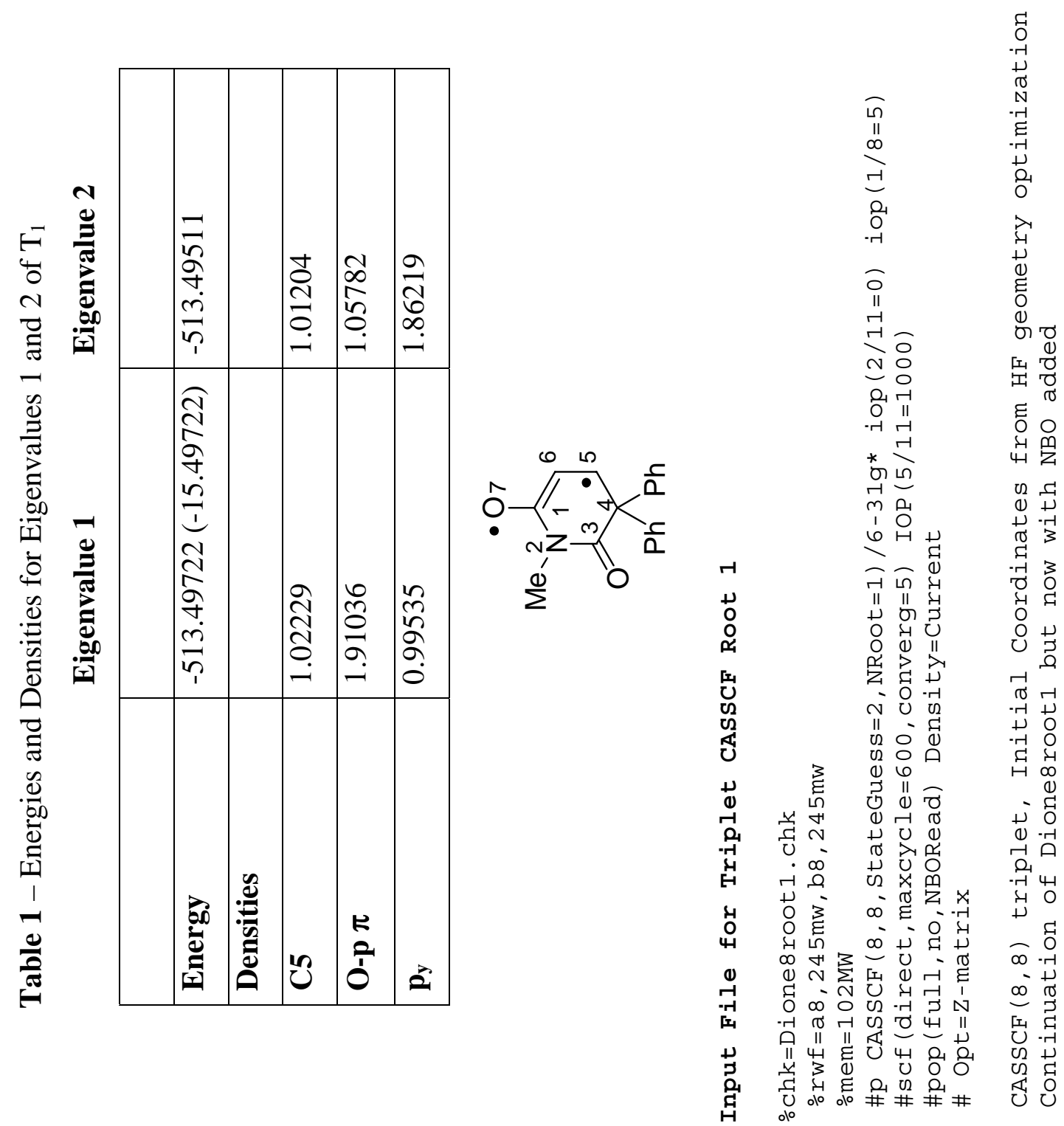


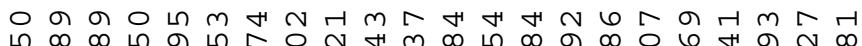

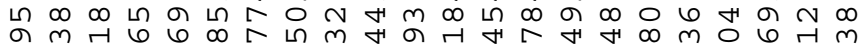

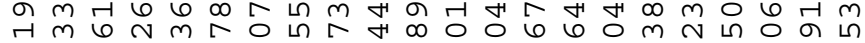

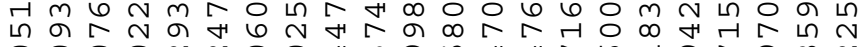

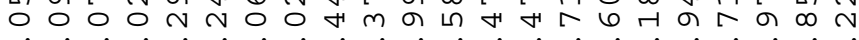

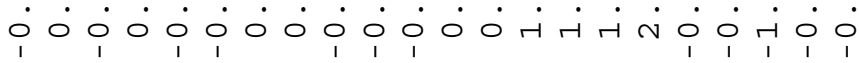

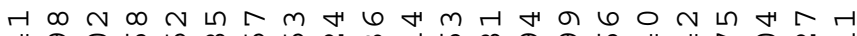
अ

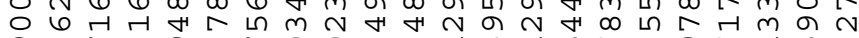

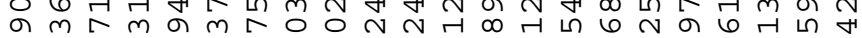

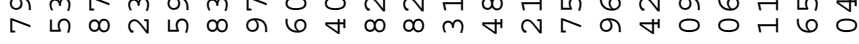

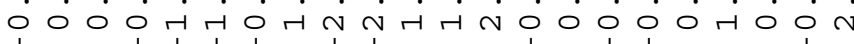

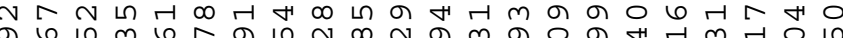

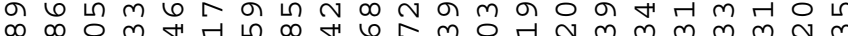

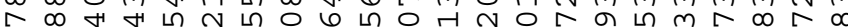

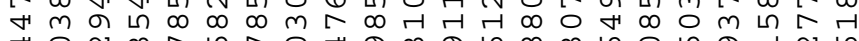

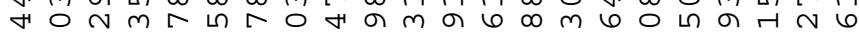

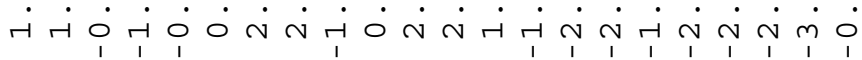

m

○U年UUUU○U出出出出出U出出出U出出出 


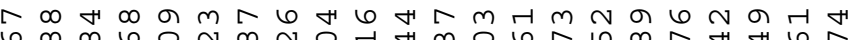

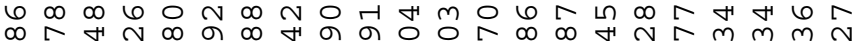

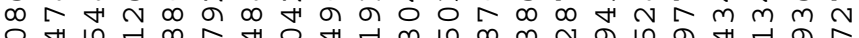

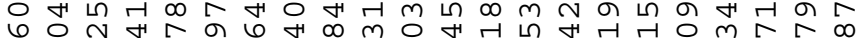

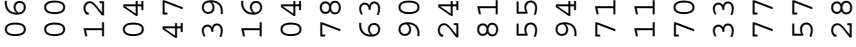

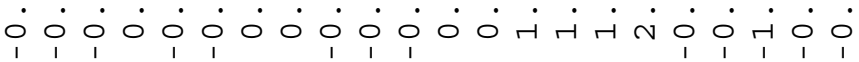

+
0
0
م

焉

Ư

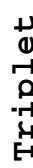



$\underset{-1}{-1}$

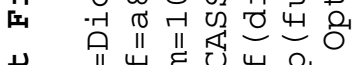

\II

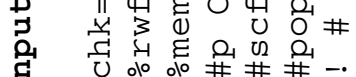

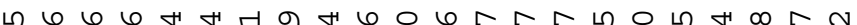

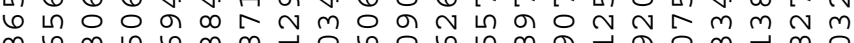
m

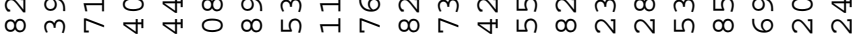

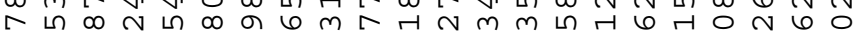

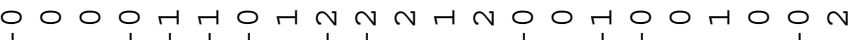

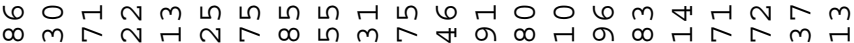

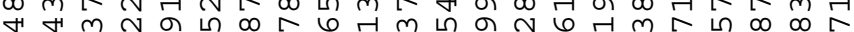

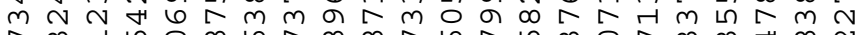

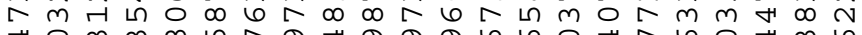

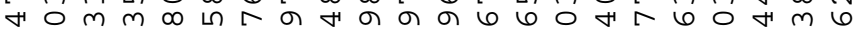

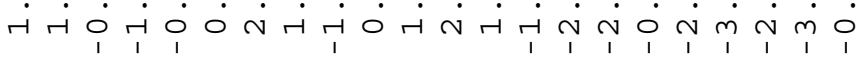
m

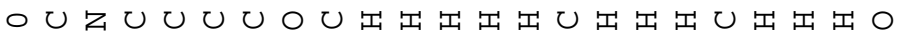

$\circ 0$ $\dot{\sim} \dot{\sim}$ $\therefore$ $\dot{0} \dot{0}$ $\therefore$ : 00 : : 00 i i त 00 N N 다 00 i i 다 다 $\circ 0$ N N 다 00 


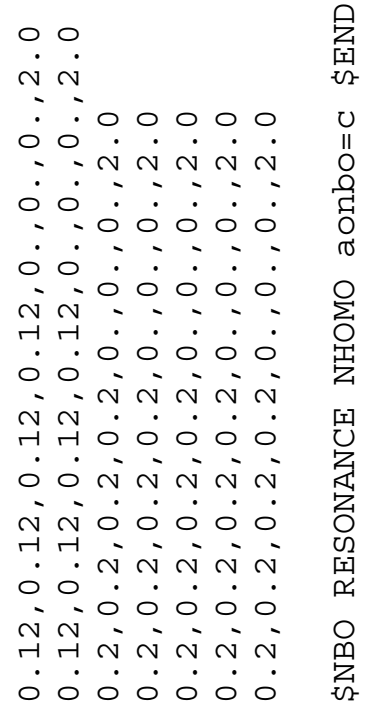

